\title{
Retracted: Plasmonic and Thermooptical Properties of Spherical Metallic Nanoparticles for Their Thermoplasmonic and Photonic Applications
}

\author{
Journal of Nanoparticles \\ Received 16 December 2015; Accepted 16 December 2015 \\ Copyright (C) 2015 Journal of Nanoparticles. This is an open access article distributed under the Creative Commons Attribution \\ License, which permits unrestricted use, distribution, and reproduction in any medium, provided the original work is properly \\ cited.
}

The paper titled "Plasmonic and Thermooptical Properties of Spherical Metallic Nanoparticles for Their Thermoplasmonic and Photonic Applications" [1], published in Journal of Nanoparticles, has been retracted as it was accepted for publication on the basis of peer review reports that were submitted from fraudulent reviewer accounts.

In late 2014, a number of publishers discovered widespread abuse of the peer review process, including cases of identity theft and faked review reports. In July 2015, Hindawi concluded an extensive investigation into peer review fraud and identified a number of articles that had been accepted on the basis of fraudulent peer review reports. In accordance with the recommendations of the Committee on Publication Ethics (COPE), Hindawi sent these manuscripts for re-review using independent Editorial Board Members. Following this re-review process, this article has been retracted as it was deemed unsuitable for publication.

\section{References}

[1] V. K. Pustovalov, L. G. Astafyeva, and W. Fritzsche, "Plasmonic and thermooptical properties of spherical metallic nanoparticles for their thermoplasmonic and photonic applications," Journal of Nanoparticles, vol. 2014, Article ID 893459, 15 pages, 2014. 


\author{
Victor K. Pustovalov, ${ }^{1}$ Liudmila G. Astafyeva, ${ }^{2}$ and Wolfgang Fritzsche ${ }^{3}$ \\ ${ }^{1}$ Belarusian National Technical University, Nezavisimosti Prospect 65, 220013 Minsk, Belarus \\ ${ }^{2}$ B.I. Stepanov Institute of Physics, National Academy of Sciences of Belarus, Nezavisimosti Prospect 68, 220072 Minsk, Belarus \\ ${ }^{3}$ Leibniz Institute of Photonic Technology, 07702 Jena, Germany \\ Correspondence should be addressed to Victor K. Pustovalov; pustovalovv@mail.ru \\ and Wolfgang Fritzsche; fritzsche@ipht-jena.de \\ Received 15 July 2014; Accepted 3 August 2014; Published 14 October 2014
}

Academic Editor: Amir Kajbafvala

Copyright (C 2014 Victor K. Pustovalov et al. This is an open access article distributed under the Creative Commons Attribution License, which permits unrestricted use, distribution, and reproduction in any medium, provided the original work is properly cited.

Investigations and use of nanoparticles (NPs) as photothermal (PT) agents in laser and optical nanotechnology are fast growing areas of research and applications. The potential benefits of NPs applications include possibility for thermal imaging and treatment of materials containing of NPs, applications of NPs for light-to-thermal energy conversion, in catalysis, laser nanomedicine, and chemistry. Efficiency of applications of metallic NPs for laser and optical nanotechnology depends on plasmonic and thermophysical properties of NPs, characteristics of radiation, and surrounding medium. Here we present the results of comparative analysis of NP properties (plasmonic, thermooptical, and others) allowing selecting their parameters for thermoplasmonic and photonic applications. Plasmonic and thermooptical properties of several metallic (aurum, silver, platinum, cobalt, zinc, nickel, titanium, cuprum, aluminum, molybdenum, vanadium, and palladium) NPs are theoretically investigated and analysis of them is carried out. Investigation of the influence of NPs parameters (type of metal, radii, optical indexes, density, and heat capacity of NP material), characteristics of radiation (wavelength and pulse duration), and ambient parameters on plasmonic and thermophysical properties of NPs has been carried out. It was established that maximum value of thermooptical parameter (maximum NP temperature) can be achieved with the use of absorption efficiency factor of NP smaller than its maximum value.

\section{Introduction}

Recent advances in photothermal nanotechnology based on the use of nanoparticles (NPs) and optical (laser) radiation have been demonstrated for their great potential. In recent years, the laser-NP interaction, absorption, and scattering of radiation energy by NP have become of great interest and an increasingly important for topic in photonic and laser nanotechnology [1-27] (also see the references in these papers). There are many reasons for this interest including application of NPs in different fields, such as catalysis $[1,2]$, laser nanobiomedicine [3-11], nanooptics and nanoelectronics [12-15], laser processing of metallic NPs in nanotechnology [16-23], and light-to-heat conversion [24-27].
Most of these technologies rely on the position and strength of the surface plasmon on a nanosphere and the fact that NP will absorb and scatter radiation energy well at resonance wavelength. Successful applications of NPs in photonics and thermoplasmonics are based on appropriate plasmonic and optical properties of NPs. High absorption of radiation by NPs can be used for conversion of absorbed energy into NP thermal energy, heating of NP itself and ambient medium, and following photothermal phenomena in laser and optical nanotechnology and nanomedicine. High scattering of radiation is essential for optical diagnostics and imaging applications based on light scattering.

Metallic NPs are mostly interesting for different nanotechnologies among other NPs. First investigations of optical 
properties of metallic NPs were carried out in $[28,29]$. The attempts to search for the "ideal" plasmonic NPs were carried out in many papers. Optical absorption efficiency of some metallic NPs was investigated in [28-33]. Thermooptical analysis and selection of the properties of gold NPs for laser applications in nanotechnology were carried out in $[8,26,34]$. Searching for better plasmonic materials (metals) was carried out in $[13,32,35,36]$ based on investigations of quality factors of each metal. Different metallic NPs (gold, silver, platinum, zinc, etc.) were used in [1-36]. Gold and silver NPs were considered as the most appropriate ones and widely used in experiments. Methods of chemical synthesis of metallic NPs have been developed and presented in [37-39].

On the other side, a comparative analysis of optimal parameters of different metallic NPs for using them as PT agents in thermoplasmonics and laser nanotechnology is still missing. Here we propose the results for analysis of the NP properties for their photonic and thermoplasmonic applications.

Plasmonic and thermooptical properties of metallic NPs were theoretically investigated and compared in this paper based on computer modeling. We carry out complex investigation of the plasmonic and thermooptical properties of spherical metallic NPs for their interaction with optical (laser) radiation placed (embedded) in some ambient medium. We investigated the influence of the parameters of radiation, NP, and ambient medium on the properties of this interaction.

\section{Plasmonic and Thermooptical Parameters of Nanoparticles}

Among different characteristics of NPs, laser radiation, and ambient medium that will determine NP plasmonic and thermooptical properties we can note the following ones:

(1) laser (optical) radiation-(a) pulse duration $t_{p}$, (b) wavelength $\lambda$, and (c) radiation (laser) exposure (energy density) $E_{0}$, intensity $I_{0}=E_{0} / t_{P}$;

(2) spherical nanoparticle-(a) type of NP metal with its values of density $\rho_{0}$, heat capacity $c_{0}$, optical indexes of refraction $n_{0 \lambda}$, and absorption $\chi_{0 \lambda}$ of NP metal and (b) NP radius $r_{0}$;

(3) nonabsorbing surrounding medium-(a) coefficient of thermal conductivity $k_{\infty}=$ const and (b) optical indexes of refraction $n_{\lambda}$.

Consider the parameters that characterize the transformation of radiation energy in the processes of NP-radiation interaction.

Efficiency factors of absorption $K_{\mathrm{abs}}$, scattering $K_{\text {sca }}$, and extinction $K_{\text {ext }}$ of radiation by NP [29] determine the optical properties of NP.
Parameter $P_{1}$ describes the correlation between absorption and scattering of radiation by NP. Parameter $P_{1}$ characterizes the contribution of the processes of absorption and scattering to the general energy balance of the NP:

$$
P_{1}=\frac{K_{\mathrm{abs}}}{K_{\mathrm{sca}}}
$$

The efficiency factor of absorption of laser radiation by NPs $K_{\text {abs }}$ can be greater or smaller than the factor of scattering of radiation by NP $K_{\text {sca }}$ in the cases of predominant role of absorption or scattering in the process of radiation interaction with NP:

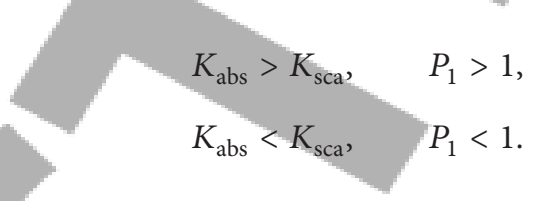

The parameter $\Delta T_{0} / E_{0}[6-8]$ can be used for determination of thermooptical properties of NPs

$$
\frac{\Delta T_{0}}{E_{0}}=\frac{K_{\mathrm{abs}} r_{0}}{4 k_{\infty} t_{P}}\left[1-\exp \left(-\frac{t_{P}}{\tau_{0}}\right)\right],
$$

$\tau_{0}=\rho_{0} c_{0} r_{0}^{2} / 3 k_{\infty}-$ characteristic time for heating and cooling of NP. This parameter determines the increase of NP temperature $\Delta T_{0}=T_{\max }-T_{\infty}$ under action of radiation energy density with value $E_{0}=1 \mathrm{~J} / \mathrm{cm}^{2}, T_{\max }$, maximum temperature of NP at $t=t_{P}$, and $T_{\infty}$, initial NP temperature.

Parameter $\Delta T_{0} / E_{0}$ (3) may be viewed as NP heating efficiency under action of radiation energy with energy density $E_{0}$. For $t_{P}<\tau_{0}$ and $t_{P}>\tau_{0}$ the parameter $\Delta T_{0} / E_{0}$ will be approximately determined by the following (see (3)):

$$
\begin{aligned}
& t_{P}<\tau_{0}, \quad \frac{\Delta T_{0}}{E_{0}} \approx \frac{3 K_{\mathrm{abs}}}{4 \rho_{0} c_{0} r_{0}}, \\
& t_{P}>\tau_{0}, \quad \frac{\Delta T_{0}}{E_{0}} \approx \frac{K_{\mathrm{abs}} r_{0}}{4 k_{\infty} t_{P}} .
\end{aligned}
$$

In general, the parameter $\Delta T_{0} / E_{0}((3),(4 \mathrm{a})$, and (4b)) depends on characteristics of radiation, $\lambda$ and $t_{P}$, metallic $\mathrm{NP}, K_{\mathrm{abs}}(\lambda), r_{0}, c_{0}$, and $\rho_{0}$, and ambient medium, $k_{\infty}$, index of medium refraction $n_{\lambda}$. Combinations $K_{\mathrm{abs}}\left(r_{0}\right) / r_{0}$ and $K_{\mathrm{abs}}\left(r_{0}\right) r_{0}$ in $(4 \mathrm{a})$ and $(4 \mathrm{~b})$ determine the range of radii $r_{0}$ appropriate for the achievement of the maximum value of $T_{0}$ under a fixed value of $\lambda, K_{\mathrm{abs}}(\lambda)$. The combination $1 / c_{0} \rho_{0}$ determines the influence of NP metal properties on the maximum value of $T_{0}$. Values of $k_{\infty}$ and $n_{\lambda}$ determine the influence of surrounding medium on thermooptical properties of NPs. The parameter of $\Delta T_{0} / E_{0}$ does not depend on parameters of radiation $\left(t_{P}\right)$ and ambience $\left(k_{\infty}\right)$ in $(4 \mathrm{a})$. The selection of mentioned parameters in (3), (4a), and (4b) can provide maximum values $\Delta T_{0}$ for concrete values of $E_{0}$.

We will investigate the influence of all characteristics of NPs, laser radiation, and ambient medium mentioned above on plasmonic and thermooptical properties of metallic NPs. Comparative analysis of the properties of metallic NPs and their efficiency for photonic applications in nanotechnology 
have to use the following set of plasmonic and thermooptical parameters of the laser-NP interaction processes:

(i) efficiency factors of absorption $K_{\text {abs }}$, scattering $K_{\text {sca }}$, and extinction $K_{\text {ext }}$ of radiation by spherical NP;

(ii) parameter of $P_{1}(1)$;

(iii) parameter $\Delta T_{0} / E_{0}((3),(4 a)$, and (4b)).

\section{Plasmonic and Thermooptical Properties of NPs}

Calculations and analysis of plasmonic and thermooptical properties of NPs have been carried out in our investigations. We numerically calculated efficiency factors of absorption $K_{\text {abs }}$, scattering $K_{\text {sca }}$, and extinction $K_{\text {ext }}$ of radiation with wavelength $\lambda$ by spherical homogeneous metallic NP based on generalized Mie theory [29]. Values of optical indexes of refraction and absorption of metals and surrounding media were used from [40-42]. After that, we use (1) and (3) for calculation of the parameters $P_{1}$ and $\Delta T_{0} / E_{0}$. All figures presented describe the dependences of efficiency factors of absorption $K_{\mathrm{abs}}$, scattering $K_{\text {sca }}$, and extinction $K_{\text {ext }}$, parameters $P_{1}$ and $\Delta T_{0} / E_{0}$ for metallic NPs on wavelength of radiation, NP radii, pulse duration $t_{P}$, and characteristics of surrounding media. Simultaneous comparative investigation of the dependences of $K_{\mathrm{abs}}, K_{\mathrm{sca}}, K_{\mathrm{ext}}$, and $\Delta T_{0} / E_{0}$ on $\lambda$, $r_{0}$, and other characteristics is very complex and hard task. We have divided this task into two steps. A first step is the calculation and investigation of the dependences of $K_{\mathrm{abs}}, K_{\mathrm{sca}}$, $K_{\text {ext }}, P_{1}$, and $\Delta T_{0} / E_{0}$ on $\lambda$ for some fixed values of $r_{0}, t_{P}$, and selected NP metal and surrounding medium. Second one is the investigation of the dependences of $K_{\mathrm{abs}}, K_{\mathrm{sca}}, K_{\mathrm{ext}}, P_{1}$, and $\Delta T_{0} / E_{0}$ on $r_{0}$ for some fixed values of $\lambda, t_{P}$, and selected NP metal and surrounding medium. This allows investigating complex task step by step and present clear dependences of $K_{\text {abs }}, K_{\text {sca }}, K_{\text {ext }}, P_{1}$, and $\Delta T_{0} / E_{0}$ on one parameter when other parameters are constant. Figures 1-7 present the dependences of $K_{\mathrm{abs}}, K_{\mathrm{sca}}, K_{\mathrm{ext}}, P_{1}$, and $\Delta T_{0} / E_{0}$ on $\lambda, r_{0}, t_{P}$, and optical indexes of metals and surrounding media.

The heat flow from NP, placed in liquids, amorphous solids, and so forth, can be well described by the diffusive heat equation, when mean free path of heat transporter (molecule, etc.) is very short, like $\sim 10^{-8} \mathrm{~cm}$ in mentioned media [43, 44], and this one is much smaller than characteristic NP radii of $r_{0} \sim 10-100 \mathrm{~nm}$. In gases at atmospheric pressure the mean free path of molecules is about $\sim 10^{-5} \mathrm{~cm}$ and diffusive heat equation can be applied to the heat exchange of NP with gaseous medium for $r_{0} \geq 100 \mathrm{~nm}$. Methods of kinetic equation or molecular dynamics should be used for the description of heat exchange of NP in this case. But during ultrashort laser pulse action with $t_{P} \sim 10^{-10}-10^{-12} \mathrm{~s}$ on NP we can neglect NP heat exchange with surrounding gas during laser action and calculate parameter $\Delta T_{0} / E_{0}$ for $t_{P}=1 \times 10^{-12} \mathrm{~s}$ using $(4 \mathrm{a})$. The dependence $\Delta T_{0} / E_{0}(\lambda)$ for $t_{P} \sim 1 \times 10^{-10} \mathrm{~s}$ practically coincides with this one for $t_{P}=$ $1 \cdot 10^{-12} \mathrm{~s}$ and only one dependence $\Delta T_{0} / E_{0}(\lambda)$ is presented in Figures 1-6 for ambient air and $t_{P}=1 \cdot 10^{-12} \mathrm{~s}$. We can note that the values of $\Delta T_{0} / E_{0}$ for $t_{P} \sim 10^{-12} \mathrm{~s}$ can be used as upper boundaries of NP heating $\left(\Delta T_{0}=T_{\max }-T_{\infty}\right)$ without NP heat exchange.

The positions of maximum values of efficiency factors of $K_{\mathrm{abs}}^{\max }, K_{\mathrm{sca}}^{\mathrm{max}}$, and $K_{\mathrm{ext}}^{\max }$ on $\lambda$ axis are denoted in Figures $1-6$ by different vertical lines and locations $\lambda_{\mathrm{abs}}^{\mathrm{max}}$ of maximum value of absorption factor $K_{\mathrm{abs}}^{\mathrm{max}}$ on axis $\lambda$ are denoted by solid lines, $K_{\text {sca }}^{\mathrm{max}}$, dashed lines, and $\lambda_{\text {sca }}^{\max }, K_{\text {ext }}^{\max }$, dashed-dotted lines, and $\lambda_{\text {ext }}^{\text {max }}$ in the case of different values of $\lambda_{\text {abs }}^{\max }, \lambda_{\text {sca }}^{\max }$, and $\lambda_{\text {ext }}^{\max }$. In the case of equal values of $\lambda_{\mathrm{abs}}^{\max }, \lambda_{\mathrm{sca}}^{\mathrm{max}}$, and $\lambda_{\mathrm{ext}}^{\max }$ the location of coincident values of $K_{\mathrm{abs}}^{\mathrm{max}}, K_{\mathrm{sca}}^{\mathrm{max}}$, and $K_{\mathrm{ext}}^{\max }$ is denoted by solid lines. In some cases additional solid lines denote the locations of the formation of new maximums of efficiency factors (see Figures 3(c), 3(g)) or the points of sharp bend of the dependence of $K_{\mathrm{abs}}$ on $\lambda$ (see Figures 4(i) and 4(j)). Horizontal dashed lines in Figures 1(d), 1(h), 1(l)-6(d), 6(h), and $6(1)$ denote the value of $P_{1}=1$.

Figures 1-3 present the dependences of efficiency factors of $K_{\mathrm{abs}}, K_{\mathrm{sca}}$, and $K_{\text {ext }}$ of radiation, parameters $\Delta T_{0} / E_{0}$ for $t_{P}=1 \cdot 10^{-8}, 1 \cdot 10^{-12} \mathrm{~s}$, and $P_{1}$ for homogeneous metallic $\mathrm{Au}$ (Figure 1), Ag (Figure 2), and Pt (Figure 3) NPs with radii $r_{0}=10,25$, and $50 \mathrm{~nm}$ on wavelengths $\lambda$. NPs are placed in silica, water, and air ambient nonabsorbing media. Optical constants (indexes of refraction $n_{\lambda}$ and absorption $\lambda$ ) are changed in the ranges for silica $n_{\lambda} \approx 1.51-1.45$, water $n_{\lambda} \approx$ $1.39-1.33$, air $n_{\lambda} \approx 1.0$, and $\varkappa_{\lambda} \approx 0$ for all ambiences with increasing wavelength $\lambda$ in the spectral interval $\sim 200-$ $1000 \mathrm{~nm}$.

The dependences of efficiency factors of $K_{\mathrm{abs}}, K_{\mathrm{sca}}$, and $K_{\text {ext }}$ on $\lambda$ for fixed values of $r_{0}$ have complicated forms. Values of $K_{\mathrm{abs}}^{\mathrm{max}}$ are placed at $\lambda_{\mathrm{abs}}^{\max } \sim 510-530 \mathrm{~nm}$ for $\mathrm{Au}$ NPs and $\lambda_{\mathrm{abs}}^{\mathrm{max}} \sim 380-410 \mathrm{~nm}$ for Ag NPs for $r_{0}=10$, 25 , and $50 \mathrm{~nm}$ and different ambiences. Consequently the absorption of radiation is determined by plasmon resonances of silver and gold NPs in the field of electromagnetic (laser) radiation. Values of $K_{\text {abs }}$ are decreased in UV and NIR spectral intervals out of plasmon wavelengths and especially for Ag NPs these values undergo sharp decrease up to $10^{2}-10^{3}$ times. We can note a slight decrease of $K_{\mathrm{abs}}$ for Au NP in the UV spectral interval in comparison with NIR spectral interval. The behavior of dependences of $K_{\text {sca }}$ on wavelength $\lambda$ is analogous for the dependence of $K_{\mathrm{abs}}(\lambda)$. Maximum value of $K_{\mathrm{sca}}^{\mathrm{max}}$ for Ag NPs achieves $K_{\mathrm{sca}} \sim 11 \div 15$ in the interval of $\lambda \sim 410 \div 430 \mathrm{~nm}$ and $r_{0}=25 \mathrm{~nm}$. Dependence of $K_{\text {ext }}$ on $\lambda$ presents itself the sum of the dependences of $K_{\mathrm{abs}}(\lambda)$ and $K_{\text {sca }}(\lambda)$. The values of $K_{\mathrm{abs}}^{\mathrm{max}}$ and $K_{\text {ext }}^{\mathrm{max}}$ for Au, Pt NPs for $r_{0}=10$ and $25 \mathrm{~nm}$ and for Ag NPs $r_{0}=10 \mathrm{~nm}$ practically coincide with each other and for different ambiences (see Figures 1-3). But for $r_{0}=50 \mathrm{~nm}$ values of $K_{\mathrm{sca}}^{\mathrm{max}}$ and $K_{\mathrm{ext}}^{\max }$ are greater than $K_{\mathrm{abs}}^{\mathrm{max}}$. This fact was also noted in [31]. An increase of $r_{0}$ may lead to increase or decrease of the maximum values of $K_{\mathrm{abs}}, K_{\mathrm{sca}}$, and $K_{\mathrm{ext}}$. Coincidence of different vertical lines in figures means the coincidence of corresponding values of optical parameters. Placements of $K_{\text {ext }}$ at $\lambda \sim 292 \mathrm{~nm}$ and $443 \mathrm{~nm}$ for NPs in water for $r_{0}=25$ and $50 \mathrm{~nm}$ quantitatively coincide with experimental data [45].

Placements of maximum values of $K_{\mathrm{abs}}, K_{\mathrm{sca}}$, and $K_{\mathrm{ext}}$ on axis $\lambda$ can be different in some cases (Figures $1(\mathrm{c}), 1(\mathrm{~g}), 1(\mathrm{k})-$ $3(\mathrm{c}), 3(\mathrm{~g})$, and $3(\mathrm{k}))$. The formation of additional maximums 


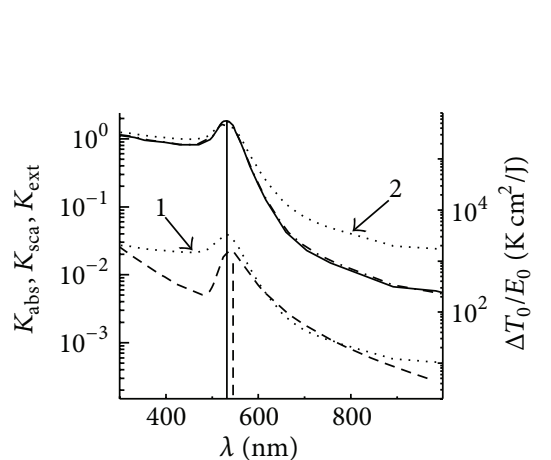

(a)

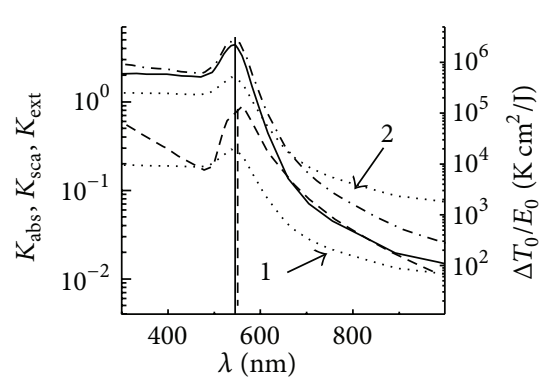

(b)

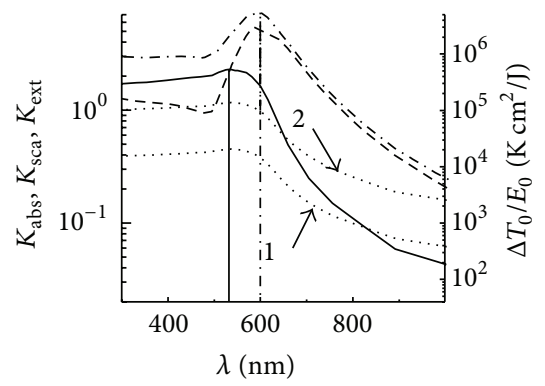

(c)

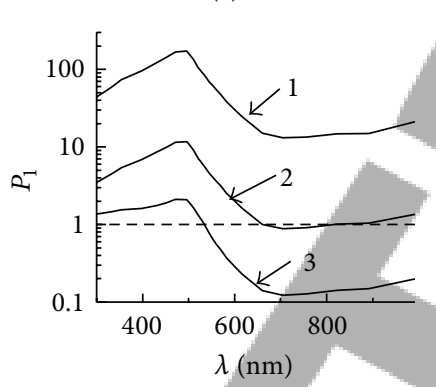

(d)

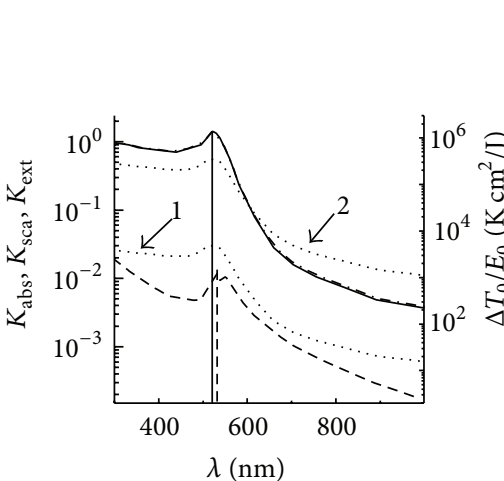

(e)

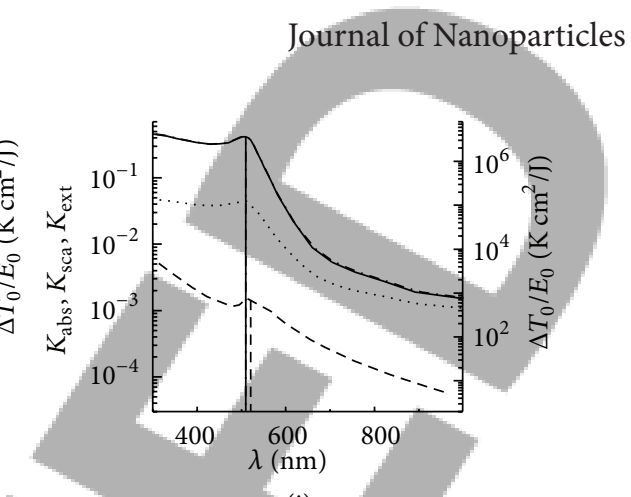

(i)
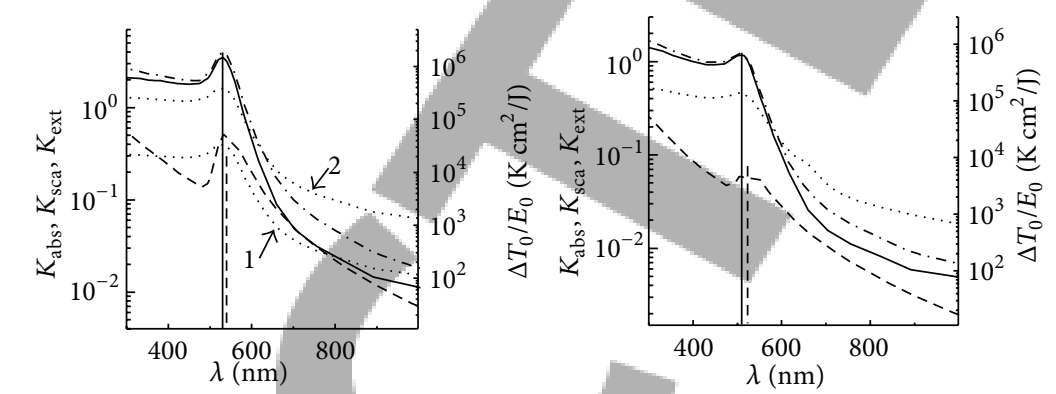

(j)

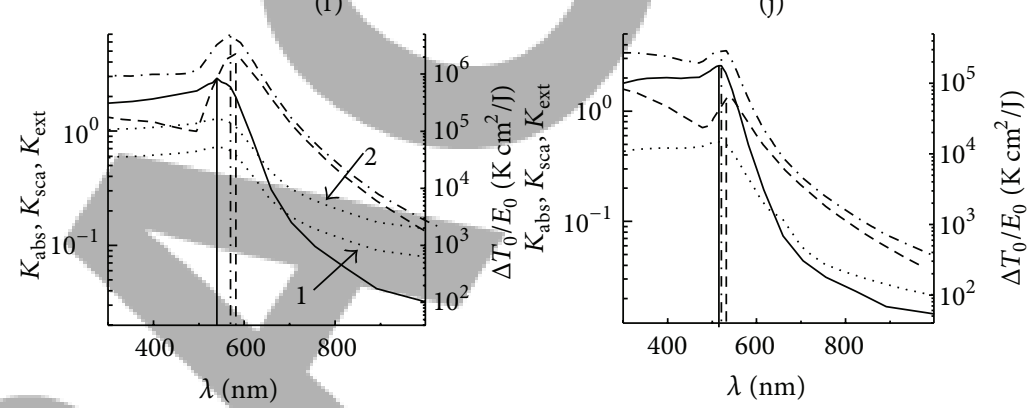

(k)

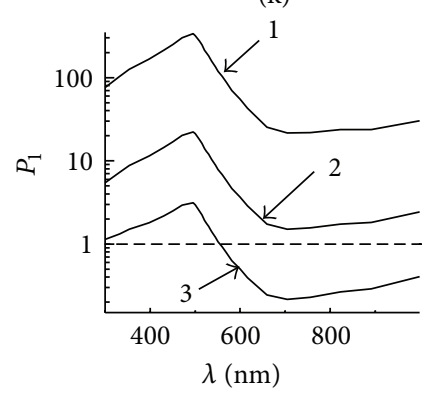

(l)

Figure 1: Dependences of efficiency factors of absorption $K_{\text {abs }}$ (solid), scattering $K_{\text {sca }}$ (dashed), and extinction $K_{\text {ext }}$ (dashed-dotted) of radiation and parameter $\Delta T_{0} / E_{0}$ (dotted) for $t_{P}=1 \cdot 10^{-8}(1), 1 \cdot 10^{-12}$ (2) s for Au NPs with radii $r_{0}=10((\mathrm{a}),(\mathrm{e})$, and (i)), 25 ((b), $(\mathrm{f})$, and $(\mathrm{j}))$, and $50((\mathrm{c}),(\mathrm{g})$, and $(\mathrm{k})) \mathrm{nm}$, and the dependences of parameter $P_{1}\left((\mathrm{~d}),(\mathrm{h})\right.$, and (l)) for Au NPs with radii $r_{0}=10 \mathrm{~nm}(1)$, $r_{0}=25 \mathrm{~nm}(2)$, and $r_{0}=50 \mathrm{~nm}$ (3) on wavelengths $\lambda$. Au NPs are placed in silica ((a)-(d)), water ((e)-(h)), and air ((i)-(l)).

of $K_{\mathrm{abs}}, K_{\mathrm{sca}}$, and $K_{\mathrm{ext}}$ on axis $\lambda$ can be connected with possible manifestation of resonances with higher orders (Figures 3(c) and $3(\mathrm{~g})$ Pt NPs). An increase of NP radii to $r_{0}=50 \mathrm{~nm}$ shifts the maximum values of $K_{\mathrm{sca}}$ and $K_{\text {ext }}$ in the region of greater values of $\lambda$. The value of shift of $\Delta \lambda_{\max }=\lambda_{\max }\left(K_{\mathrm{abs}}\right)-$ $\lambda_{\text {max }}\left(K_{\text {sca }}\right)$ increases with increasing of $r_{0}$, for $r_{0}=10 \mathrm{~nm}$ $\Delta \lambda_{\max } \sim 5-13 \mathrm{~nm}$ for all surroundings, and for $r_{0}=50 \mathrm{~nm}$ this one achieves values of $\Delta \lambda_{\max } \sim 15-70 \mathrm{~nm}$. These values increase with increasing of $n_{\lambda}$ from air to silica for $\mathrm{Au}, \mathrm{Ag}, \mathrm{Pt}$ NPs. But $K_{\text {sca }}^{\max }$ and $K_{\text {ext }}^{\max }$ have been shifted compared to the position of $K_{\mathrm{abs}}^{\max }\left(\lambda_{\mathrm{abs}}^{\max }\right)$ up to $80-120 \mathrm{~nm}$ (Figures 1(c), 2(c), and $2(\mathrm{~g}))$ to bigger values of $\lambda$ with increasing of $r_{0}$.

Figure 3 shows for Pt NPs that maximums of absorption $K_{\mathrm{abs}}^{\max }$ and scattering $K_{\text {sca }}^{\max }$ for $r_{0}=10 \mathrm{~nm}$ are accordingly situated at wavelengths $\lambda_{\text {abs }}^{\max }=248 \mathrm{~nm}$ and $\lambda_{\text {sca }}^{\max }=150 \mathrm{~nm}$ in silica. Increase of $r_{0}$ leads to shifting of $K_{\mathrm{abs}}^{\mathrm{max}}, K_{\mathrm{sca}}^{\mathrm{max}}$, and 


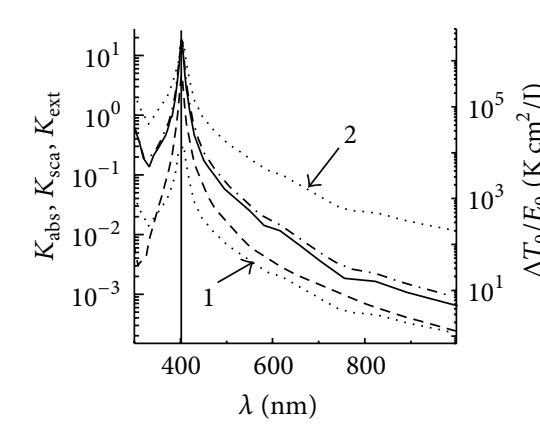

(a)

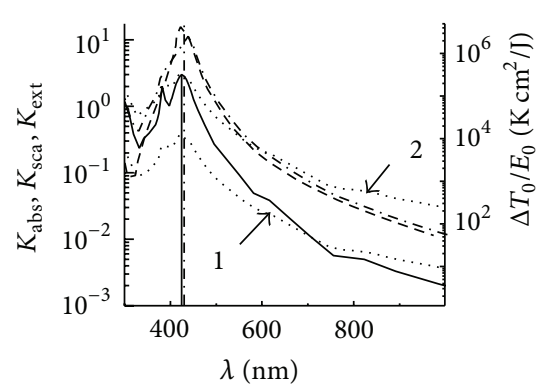

(b)

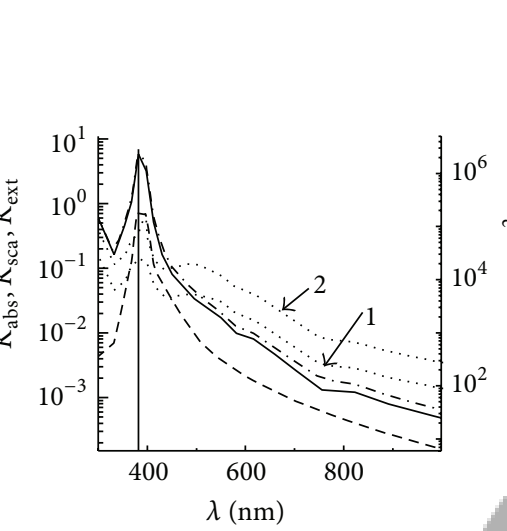

(e)
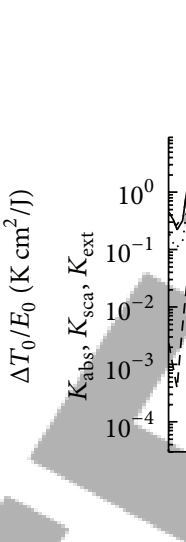

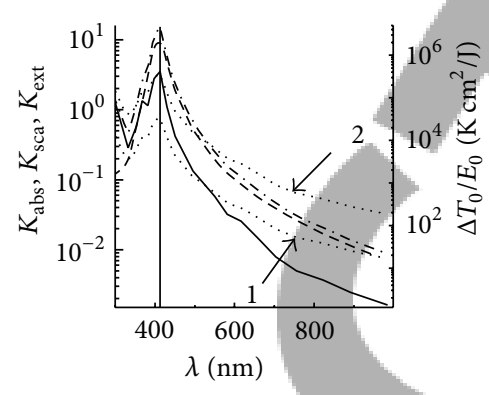

(f)

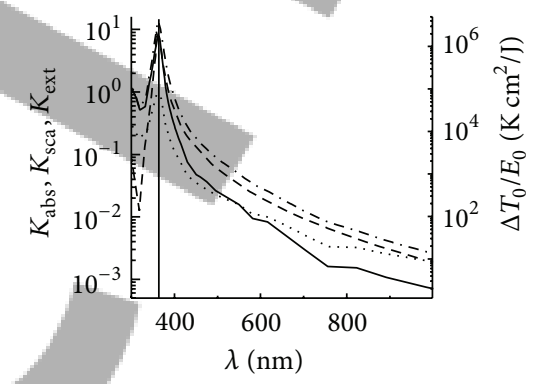

(j)

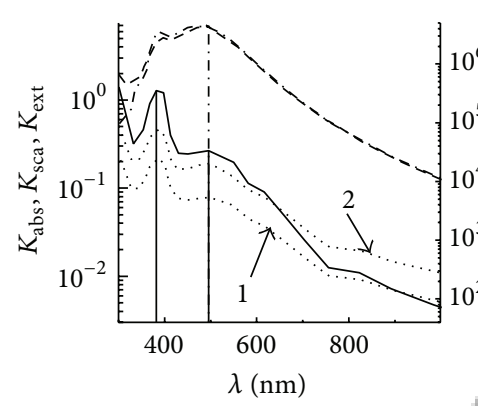

(c)

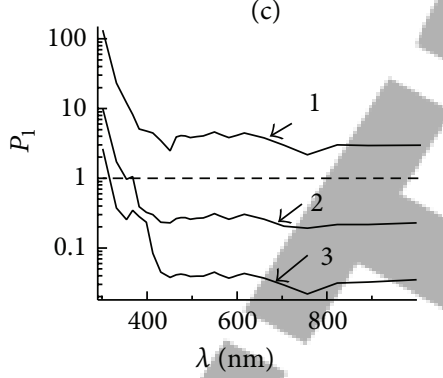

(d)

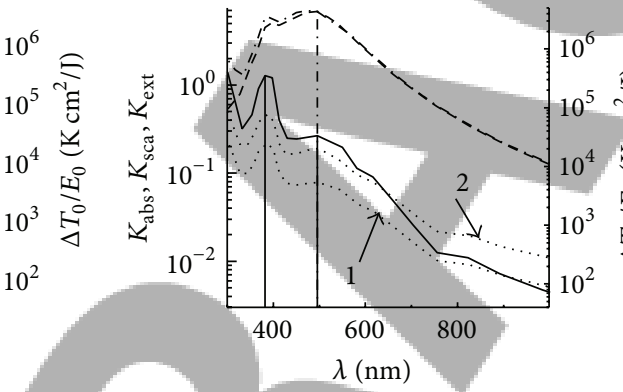

(g)

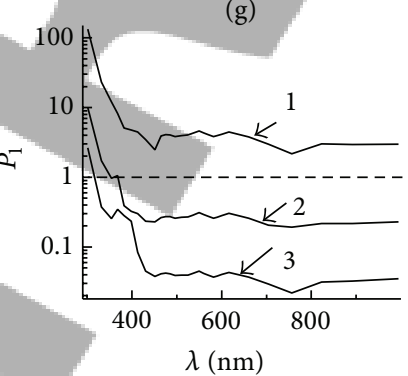

(h)

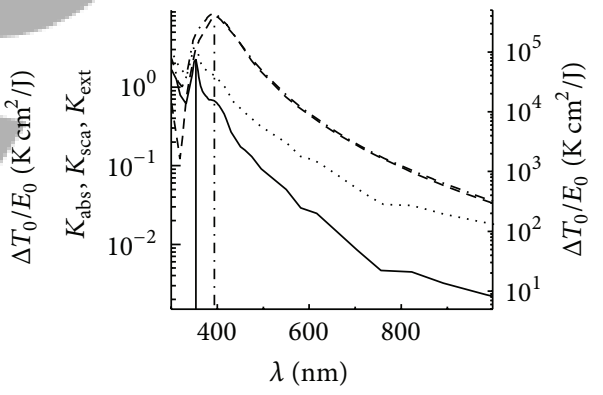

(k)

FIGURE 2: Dependences of $K_{\text {abs }}$ (solid), $K_{\text {sca }}$ (dashed), and $K_{\text {ext }}$ (dashed-dotted) of radiation and parameter $\Delta T_{0} / E_{0}\left(\right.$ dotted) for $t_{P}=1 \cdot 10^{-8}$ (1), $1 \cdot 10^{-12}(2) \mathrm{s}$ for Ag NPs with radii $r_{0}=10((\mathrm{a}),(\mathrm{e})$, and (i)), $25((\mathrm{~b}),(\mathrm{f})$, and (j)), and $50((\mathrm{c}),(\mathrm{g})$, and (k)) nm, and the dependences of parameter $P_{1}\left((\mathrm{~d}),(\mathrm{h})\right.$, and (l)) for Ag NPs with radii $r_{0}=10 \mathrm{~nm}(1), r_{0}=25 \mathrm{~nm}(2)$, and $r_{0}=50 \mathrm{~nm}(3)$ on wavelengths $\lambda$. Ag NPs are placed in silica $((\mathrm{a})-(\mathrm{d}))$, water $((\mathrm{e})-(\mathrm{h}))$, and air $((\mathrm{i})-(\mathrm{l}))$.

$K_{\text {ext }}^{\max }$ to bigger values of $\lambda$; for example, $K_{\mathrm{abs}}^{\mathrm{max}}$ is shifted from $\lambda_{\text {abs }}^{\text {max }} \sim 220-250 \mathrm{~nm}$ for $r_{0}=10 \mathrm{~nm}$ to $\lambda_{\text {abs }}^{\text {max }} \sim 450-480 \mathrm{~nm}$ for $r_{0}=50 \mathrm{~nm}$ in silica and water. But for air this shift is from $\lambda_{\text {abs }}^{\max } \sim 220 \mathrm{~nm}$ for $r_{0}=10 \mathrm{~nm}$ to $\lambda_{\text {abs }}^{\max } \sim 310 \mathrm{~nm}$ for $r_{0}=50 \mathrm{~nm}$. Maximum values of $K_{\mathrm{abs}}^{\mathrm{max}}, K_{\mathrm{sca}}^{\mathrm{max}}$, and $K_{\mathrm{ext}}^{\max }$ are at the values of $\lambda_{\text {abs }}^{\max }, \lambda_{\mathrm{sca}}^{\max }$, and $\lambda_{\text {ext }}^{\max }$ for $r_{0}=50 \mathrm{~nm}$. Moreover two maximum values of $K_{\mathrm{abs}}^{\max }$ are formed for $r_{0}=50 \mathrm{~nm}$ in silica at $\lambda_{\mathrm{abs}}^{\max }=317.9$ and $495.9 \mathrm{~nm}$ and in water at $\lambda_{\mathrm{abs}}^{\max }=290$ and $477 \mathrm{~nm}$.

For Au NPs, dependences of $P_{1}$ on $\lambda$ are determined by the dependences of $K_{\mathrm{abs}}$ and $K_{\mathrm{sca}}$ on $\lambda$. Increase of $r_{0}$ from $r_{0}=10 \mathrm{~nm}$ to $r_{0}=50 \mathrm{~nm}$ leads to a decrease of the parameter $P_{1}$ from the values of about $P_{1} \sim 20-300$ for $\lambda \sim 300-1000 \mathrm{~nm}$ up to values of about $P_{1} \sim 0.1-0.3$ for $\lambda \sim 600-1000 \mathrm{~nm}$. 


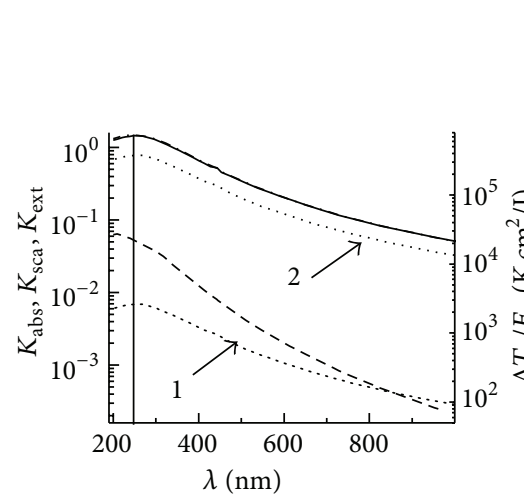

(a)

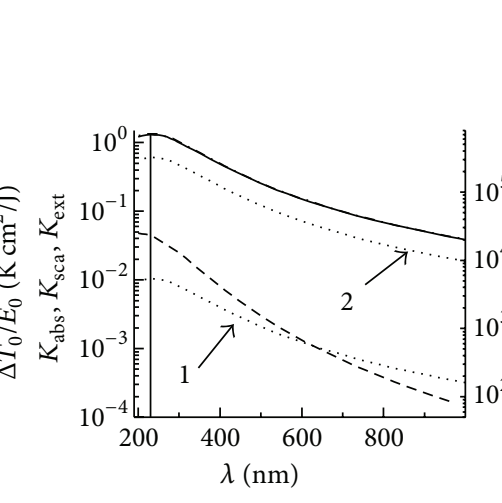

(e)

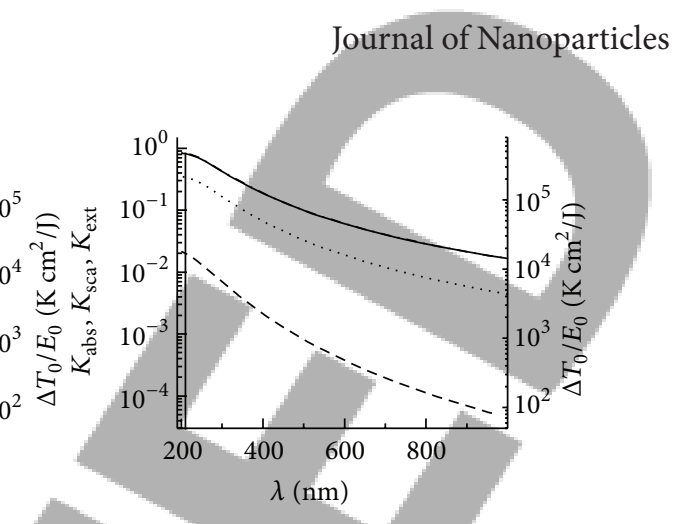

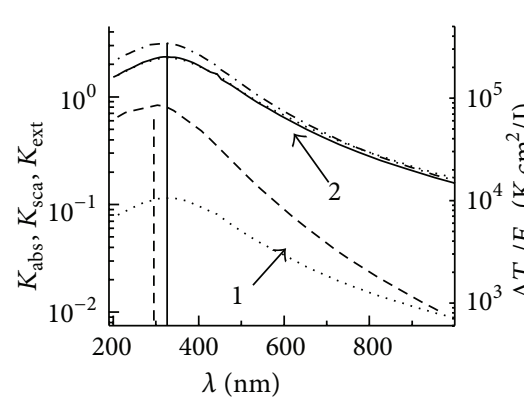

(b)

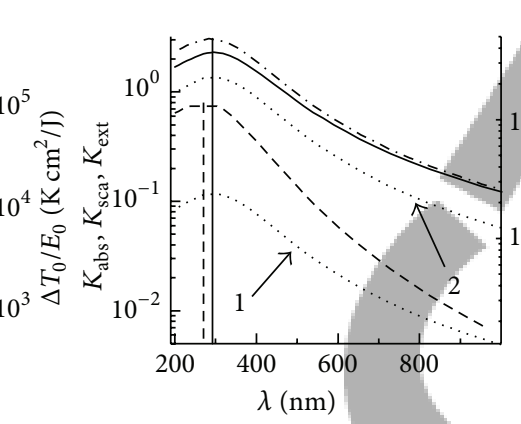

(f)

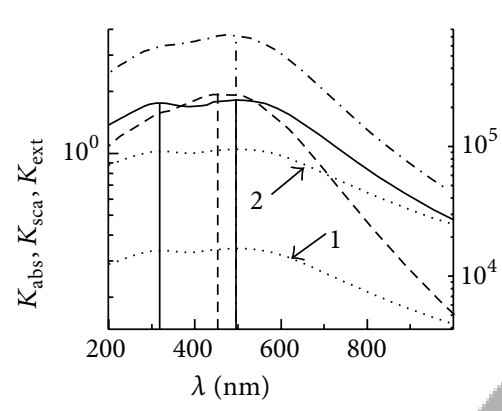

(c)

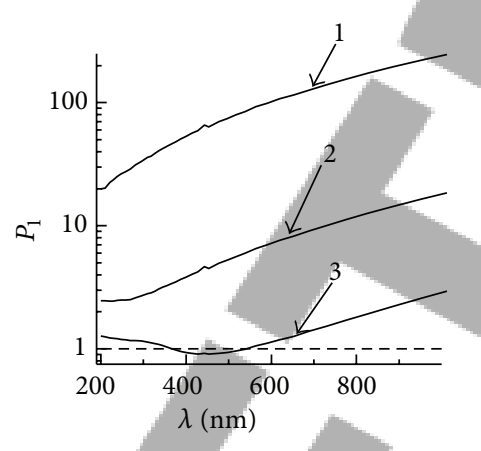

(d)
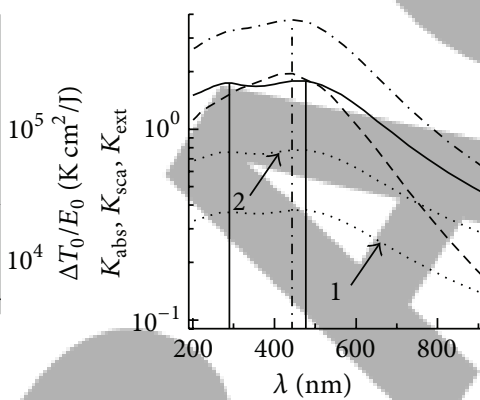

(g)

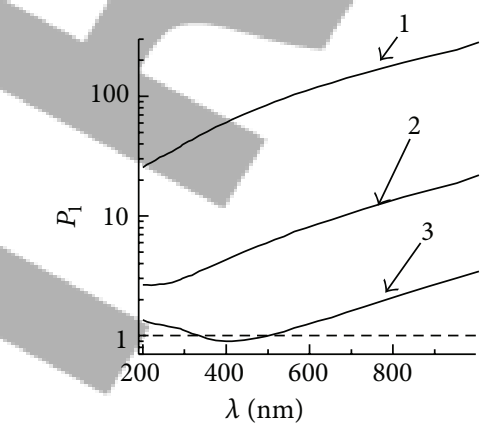

(h)

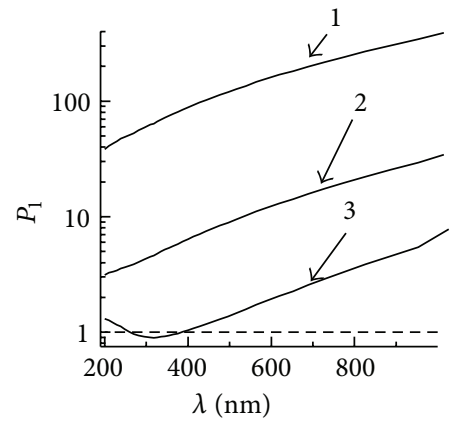

(l)

FIGURE 3: Dependences of $K_{\text {abs }}$ (solid), $K_{\text {sca }}$ (dashed), and $K_{\text {ext }}$ (dashed-dotted) of radiation and parameter $\Delta T_{0} / E_{0}\left(\right.$ dotted) for $t_{P}=1 \cdot 10^{-8}$ $(1), 1 \cdot 10^{-12}(2) s$ for Pt NPs with radii $r_{0}=10((\mathrm{a}),(\mathrm{e})$, and $(\mathrm{i})), 25((\mathrm{~b}),(\mathrm{f})$, and $(\mathrm{j}))$, and $50((\mathrm{c}),(\mathrm{g})$, and $(\mathrm{k}))$ nm, and the dependences of parameter $P_{1}\left((\mathrm{~d}),(\mathrm{h})\right.$, and (l)) for Pt NPs with radii $r_{0}=10 \mathrm{~nm}(1), r_{0}=25 \mathrm{~nm}(2)$, and $r_{0}=50 \mathrm{~nm}(3)$ on wavelengths $\lambda$. Pt NPs are placed in silica $((\mathrm{a})-(\mathrm{d}))$, water $((\mathrm{e})-(\mathrm{h}))$, and air $((\mathrm{i})-(\mathrm{l}))$.

It means sharp increase of radiation scattering by NPs with an increase of NP $r_{0}$. For Ag NPs, a sharp decrease with increasing of $\lambda$ in the spectral interval $\lambda \sim 300-400 \mathrm{~nm}$ is observed and is approximately constant in the interval $\lambda \sim$ $300-1000 \mathrm{~nm}$. General feature for all presented dependences of $P_{1}\left(\lambda, r_{0}\right)$ is the decrease of $P_{1}$ with increasing of $r_{0}$ for the whole spectral interval $\lambda \sim 200-1000 \mathrm{~nm}$.

The dependences of $P_{1}$ on $\lambda$ for Pt NPs increase with increasing $\lambda$ for $r_{0}=10$ and $25 \mathrm{~nm}$ and achieve values of $P_{1} \sim 3-200$ for the whole spectral interval because of 


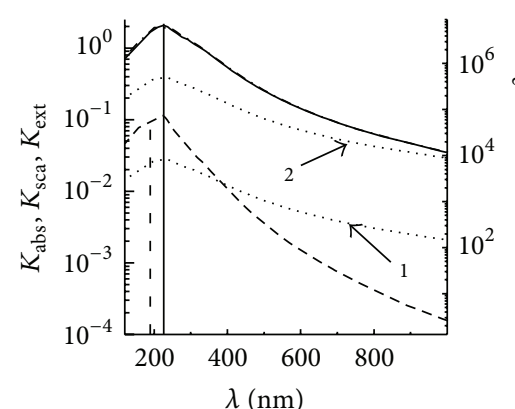

(a)

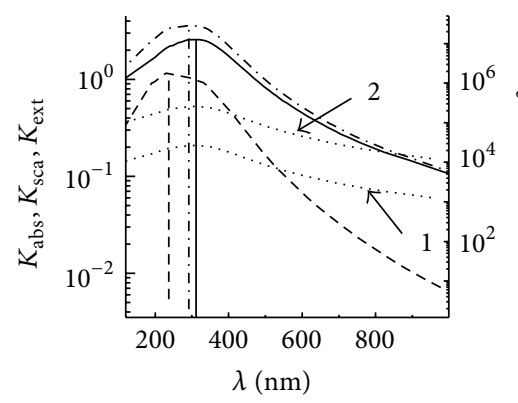

(b)

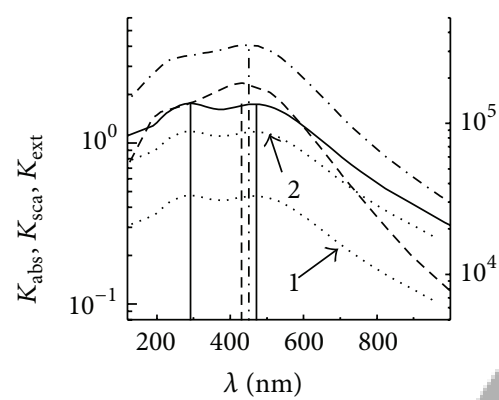

(c)

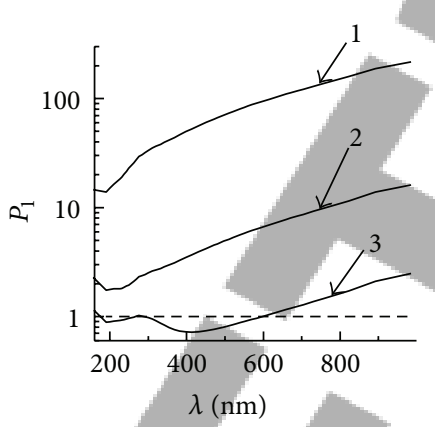

(d)

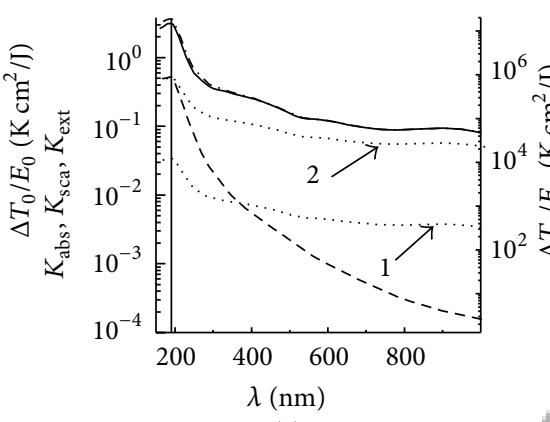

(e)
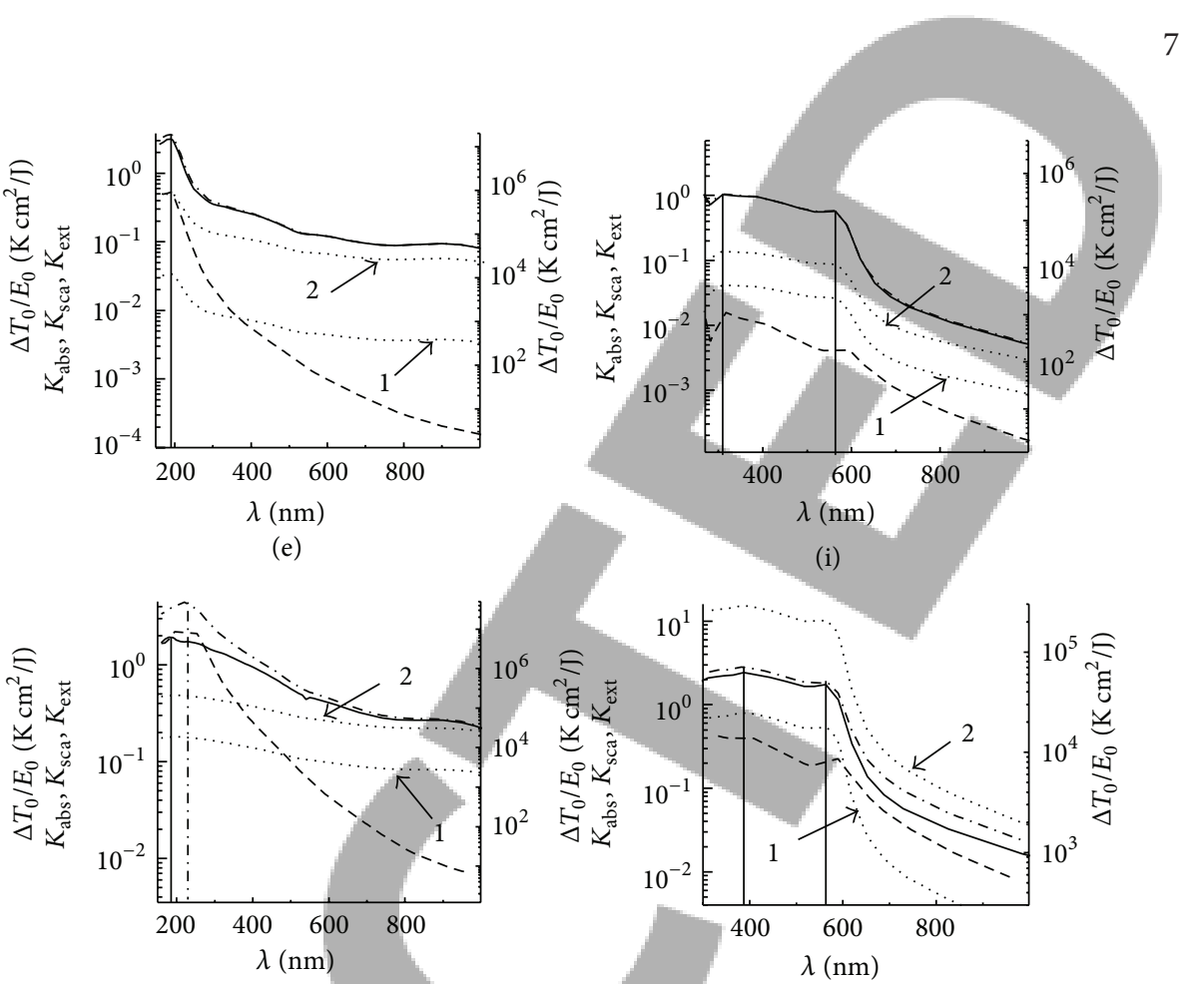

(f)

(j)

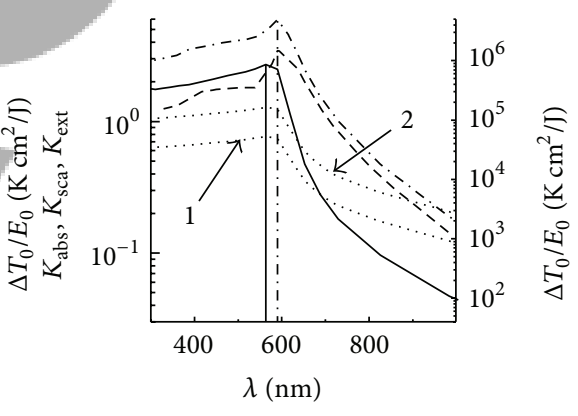

(k)

(g)

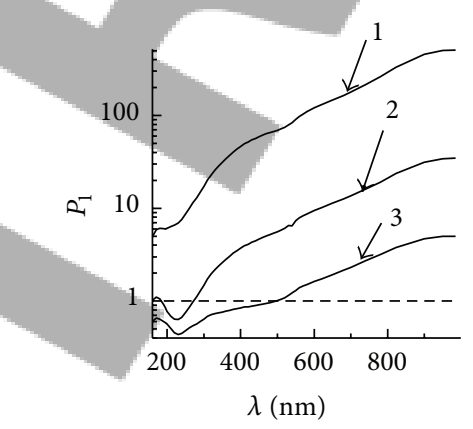

(h)

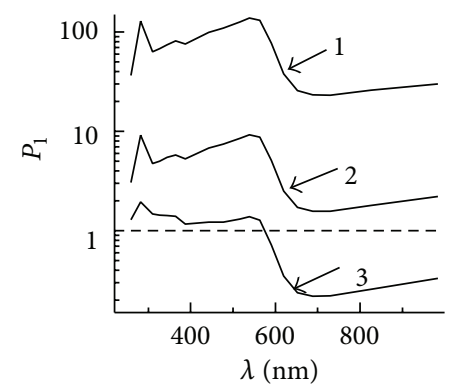

(1)

FIGURE 4: Dependences of factors $K_{\text {abs }}$ (solid), $K_{\text {sca }}$ (dashed), and $K_{\text {ext }}$ (dashed-dotted) of radiation for homogeneous metallic Pd ((a), (b), $(\mathrm{c})$, and (d)), Cu ((e), (f), (g), and (h)), and Mo ((i), (j), (k), and (l)) NPs, placed in water, with radii $r_{0}=10((\mathrm{a}),(\mathrm{d})$, and (g)), $25((\mathrm{~b}),(\mathrm{e})$, and (h)), and $50\left((\mathrm{c}),(\mathrm{f})\right.$, and (i)) $\mathrm{nm}$, parameter $\Delta T_{0} / E_{0}$ (dotted) for $t_{P}=1 \cdot 10^{-8}(1), 1 \cdot 10^{-12}(2) \mathrm{s}$, and dependences of parameter $P_{1}((\mathrm{~d})$, (h), and (1)) for 10 (1), 25 (2), and 50 (3) nm on wavelengths $\lambda$.

sharp decreasing of $K_{\text {sca }}$ with increase of $\lambda$ (see Figure 3 ). The parameter $P_{1}$ is smaller than $1, P_{1}<1$, for $r_{0}=50 \mathrm{~nm}$ and the narrow wavelength interval $\sim 350-500 \mathrm{~nm}$ for different surroundings.
Figures 1-3 describe the influence of the medium refraction indexes and thermal properties on plasmonic and thermooptical properties of $\mathrm{Au}, \mathrm{Ag}$, and Pt NPs. Concrete values of $K_{\mathrm{abs}}^{\max }$ and $k_{\infty}$ determine the influence of different 


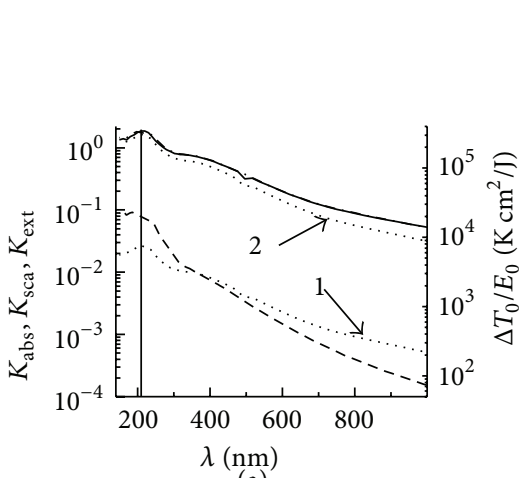

(a)

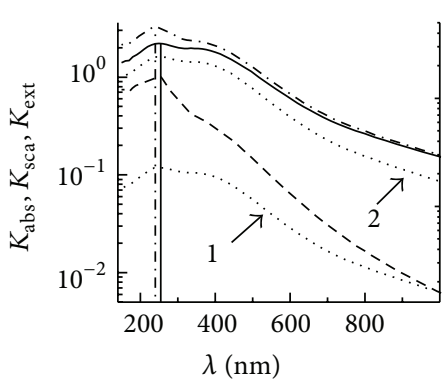

(b)

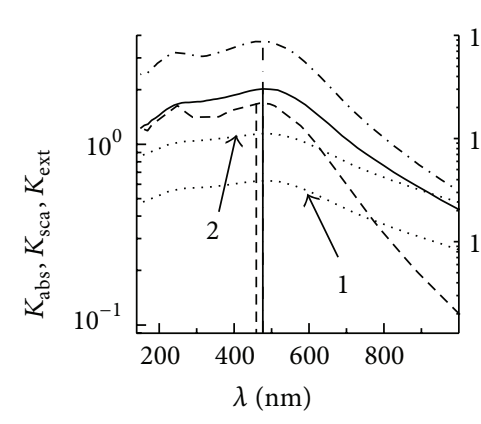

(c)

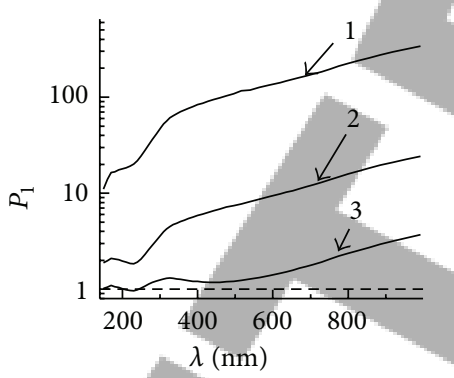

(d)

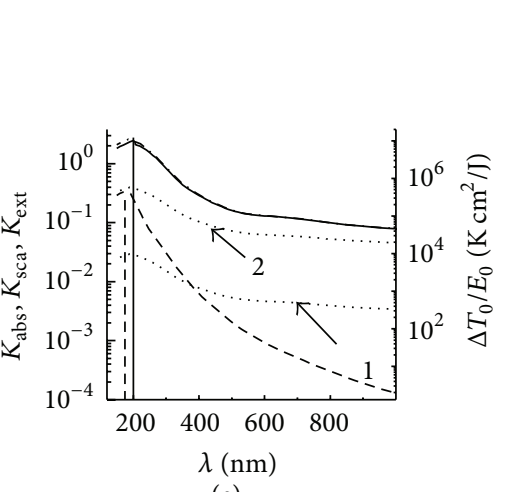

(e)
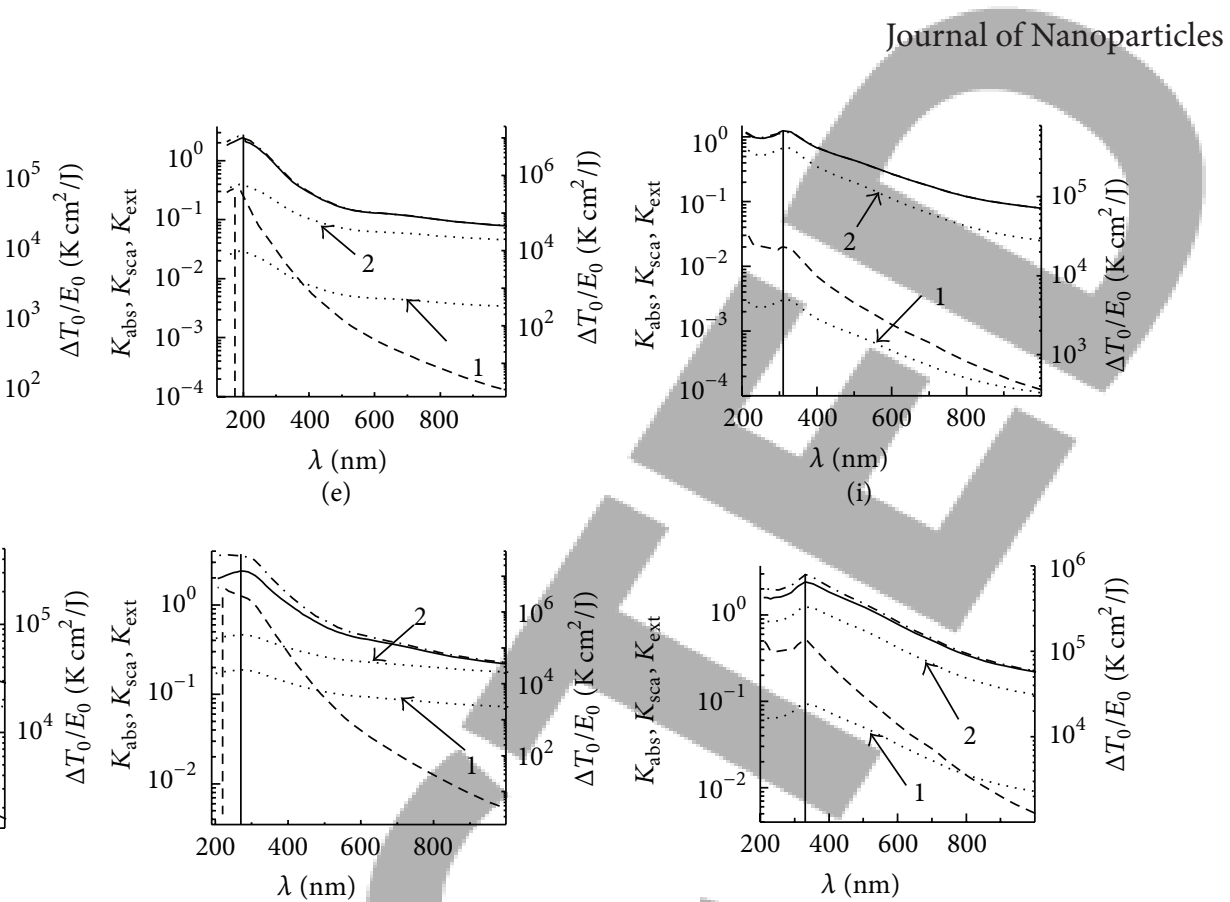

(i)

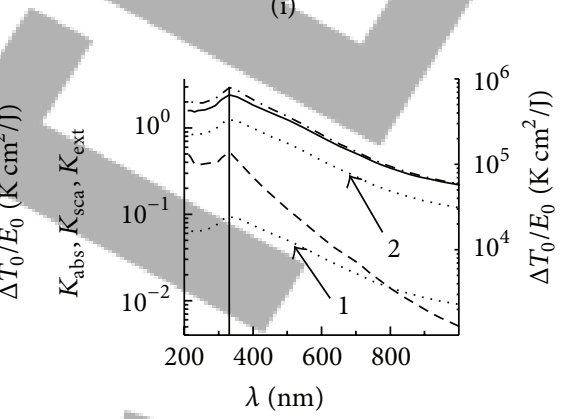

(j)

(f)

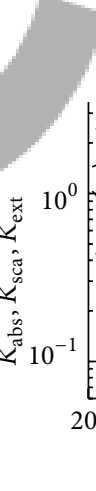

(g)

(k)

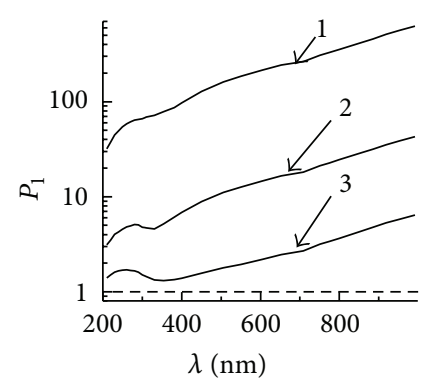

(l)

FIgURE 5: Dependences of factors $K_{\mathrm{abs}}$ (solid), $K_{\text {sca }}$ (dashed), and $K_{\text {ext }}$ (dashed-dotted) of radiation for homogeneous metallic Ni ((a), (b), $(\mathrm{c})$, and $(\mathrm{d})), \mathbf{V}((\mathrm{e}),(\mathrm{f}),(\mathrm{g})$, and $(\mathrm{h}))$, and Ti $((\mathrm{i}),(\mathrm{j}),(\mathrm{k})$, and $(\mathrm{l}))$ NPs, placed in water, with radii $r_{0}=10((\mathrm{a}),(\mathrm{d})$, and $(\mathrm{g})), 25((\mathrm{~b}),(\mathrm{e})$, and $(\mathrm{h}))$, and $50((\mathrm{c}),(\mathrm{f})$, and (i) $) \mathrm{nm}$, parameter $\Delta T_{0} / E_{0}$ (dotted) for $t_{P}=1 \cdot 10^{-8}(1), 1 \cdot 10^{-12}(2) \mathrm{s}$, and dependences of parameter $P_{1}((\mathrm{~d}),(\mathrm{h})$, and (1)) for 10 (1), 25 (2), and 50 (3) nm on wavelengths $\lambda$.

surroundings on the value of $\Delta T_{0} / E_{0}$ for different $t_{P}$ (see (3)). The wavelength shift $\Delta \lambda_{\max }$ exists between maximums of $K_{\mathrm{abs}}^{\mathrm{max}}$ from one side and maximums of $K_{\mathrm{sca}}^{\max }$ and $K_{\mathrm{ext}}^{\max }$ from a second side for silica and water. Maximum values and dependences of $K_{\mathrm{abs}}, K_{\mathrm{sca}}$, and $K_{\text {ext }}$ on wavelength are qualitatively close to each other for $r_{0}=10,25$, and $50 \mathrm{~nm}$.
The decrease of refraction index from $n_{\lambda}=1.51$ for silica to $n_{\lambda}=1.00$ for air leads to shifting of $\lambda_{\max }$ for all efficiency factors for $K_{\text {abs }}$ from $\sim 545 \mathrm{~nm}$ to $\sim 510 \mathrm{~nm}$ and for $K_{\text {sca }}$ and $K_{\text {ext }}$ from $600 \mathrm{~nm}$ to $510 \mathrm{~nm}$. Decrease of $n_{\lambda}$ for silica to $n_{\lambda}=$ 1.00 (air) leads to decrease of values of $K_{\mathrm{abs}}, K_{\mathrm{sca}}$, and $K_{\text {ext }}$ up to 4 times for $r_{0}=10$ and $25 \mathrm{~nm}$, but for $r_{0}=50 \mathrm{~nm}$ 


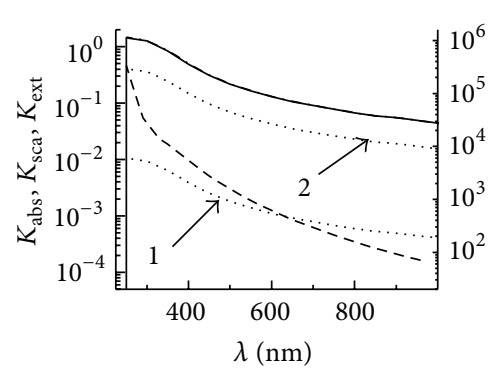

(a)

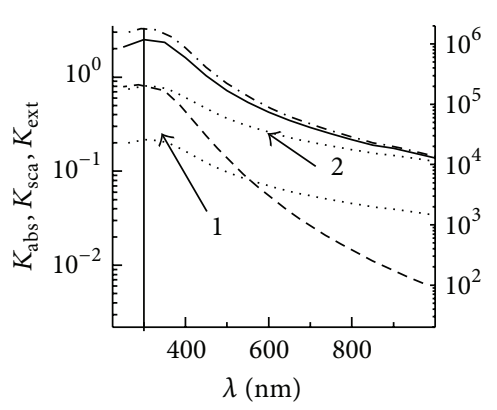

(b)

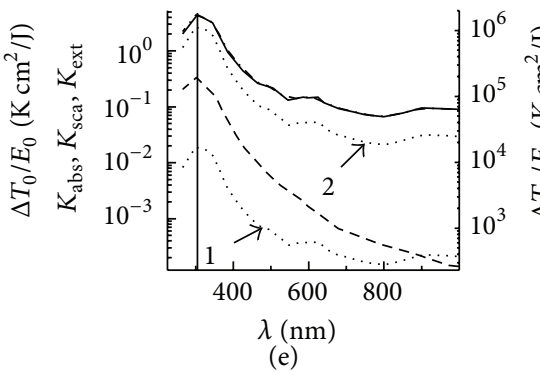

(e)
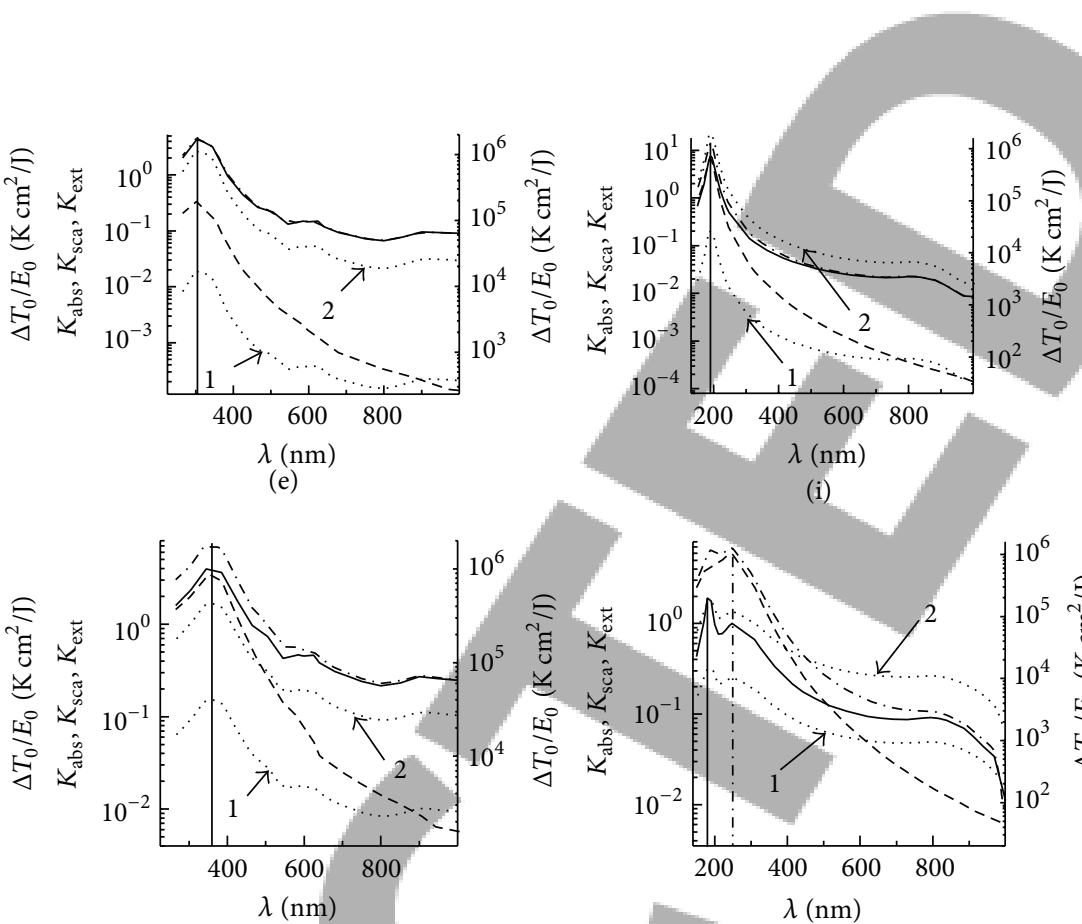

(f)

(i)

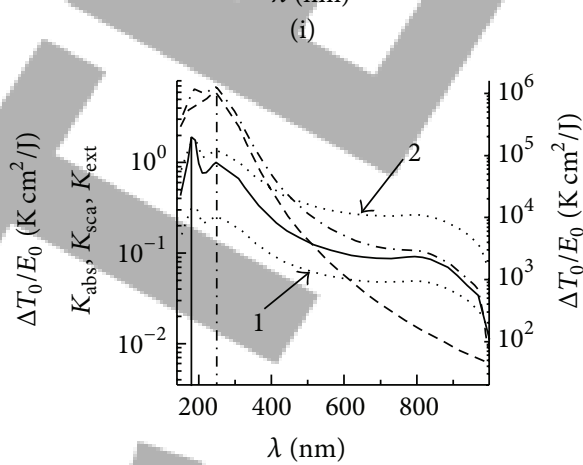

(j)

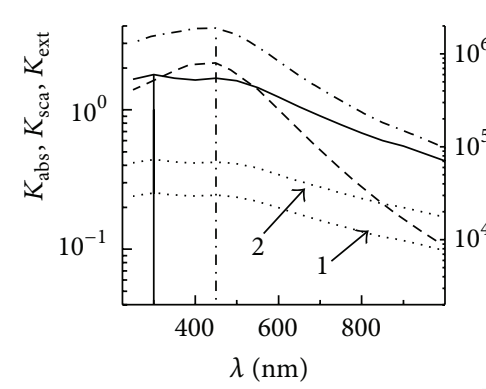

(c)

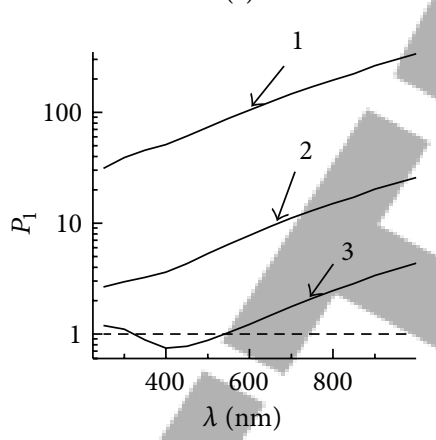

(d)

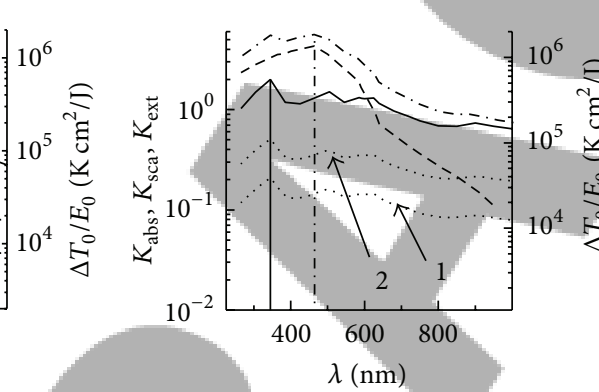

(g)

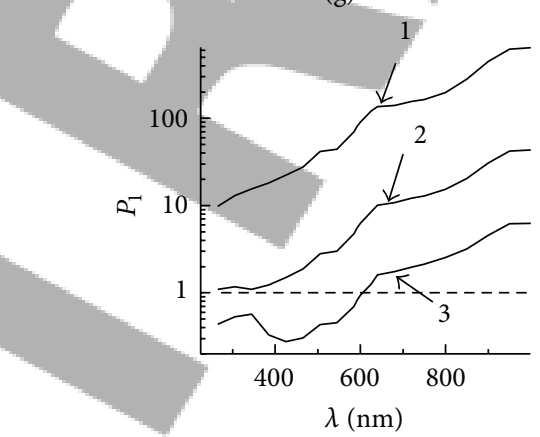

(h)

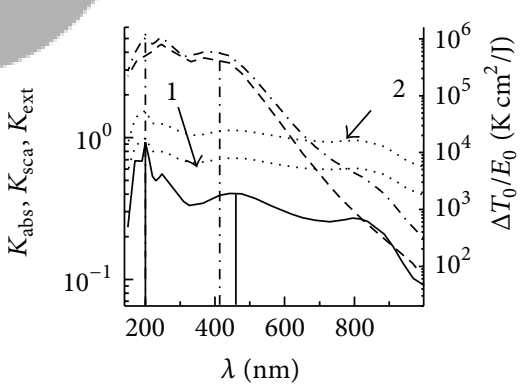

(k)

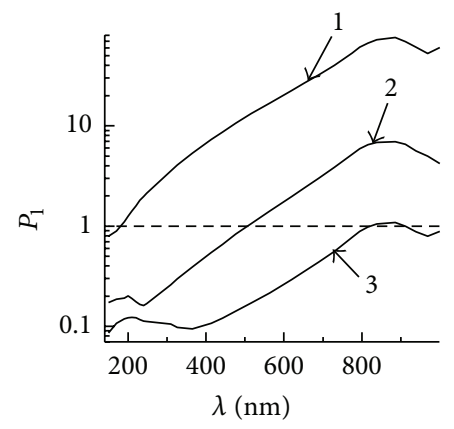

(l)

FIGURE 6: Dependences of factors $K_{\text {abs }}$ (solid), $K_{\text {sca }}$ (dashed), and $K_{\text {ext }}$ (dashed-dotted) of radiation for homogeneous metallic Co ((a), (b), $(\mathrm{c})$, and (d)), Zn ((e), (f), (g), and (h)), and Al ((i), (j), (k), and (l)) NPs, placed in water, with radii $r_{0}=10((\mathrm{a}),(\mathrm{d})$, and $(\mathrm{g})), 25((\mathrm{~b}),(\mathrm{e})$, and $(\mathrm{h})$ ), and $50\left((\mathrm{c}),(\mathrm{f})\right.$, and (i)) $\mathrm{nm}$, parameter $\Delta T_{0} / E_{0}$ (dotted) for $t_{P}=1 \cdot 10^{-8}(1), 1 \cdot 10^{-12}(2) \mathrm{s}$, and dependences of parameter $P_{1}((\mathrm{~d}),(\mathrm{h})$, and (1)) for $10(1), 25$ (2), and 50 (3) nm on wavelengths $\lambda$.

the dependence of maximum values of $K_{\mathrm{abs}}, K_{\mathrm{sca}}$ and $K_{\text {ext }}$ is rather weak and leads to the smoothing of plasmonic peaks of the dependences of $K(\lambda)$, first of all, for $r_{0}=10,25 \mathrm{~nm}$.

The spectral dependence of $\Delta T_{0} / E_{0}(\lambda)$ is determined by the dependence of $K_{\text {abs }}(\lambda)$ for all values of $r_{0}$, because of dependence $\Delta T_{0} / E_{0} \sim K_{\mathrm{abs}}(\lambda)$ in (3), (4a), and (4b). The influence of NP radius $r_{0}$ is directly realized on the value of $\Delta T_{0} / E_{0}$ and it is determined by the dependence of $K_{\mathrm{abs}}\left(r_{0}\right)$ and value of $r_{0}$ in (3), (4a), and (4b). Parameters of surroundings influence the value of $\Delta T_{0} / E_{0}$ by the value of $K_{\mathrm{abs}}\left(n_{\lambda}\right)$.

The values of $\Delta T_{0} / E_{0}$ for $t_{P}=1 \times 10^{-8} \mathrm{~s}$ are, as a rule, smaller in comparison with other ones for $t_{P}=1 \times 10^{-12} \mathrm{~s}$. It is determined by the influence of heat exchange of NP with 


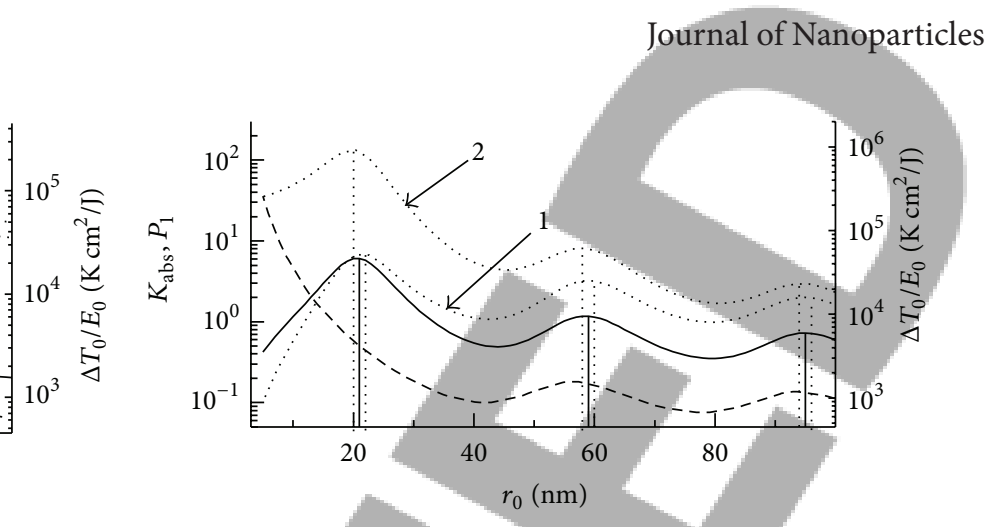

(a)

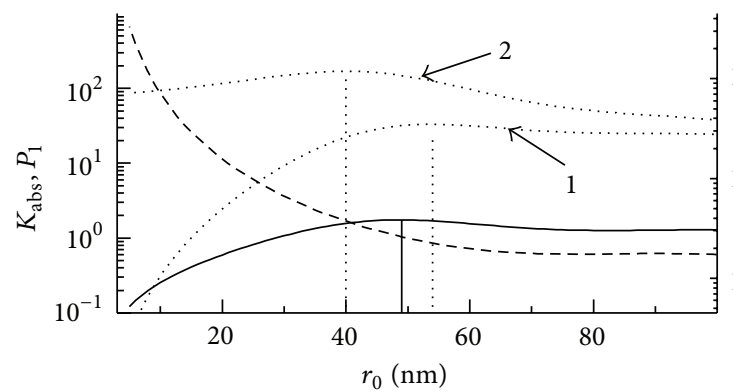

(c)

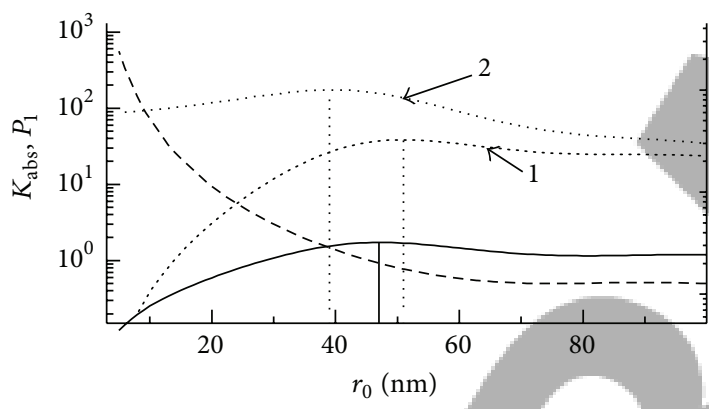

(e)

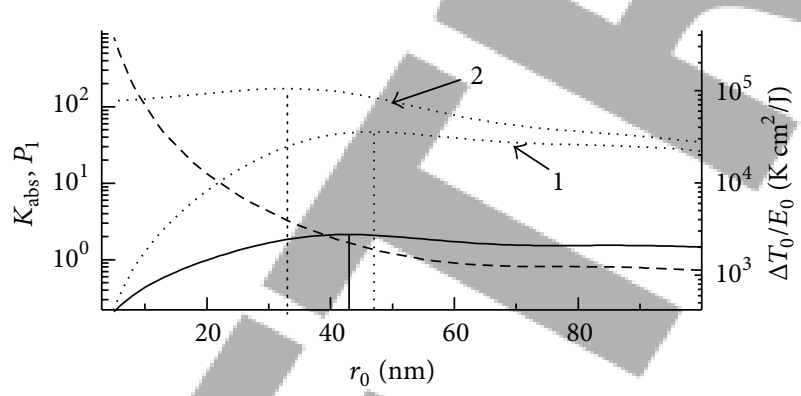

(g)

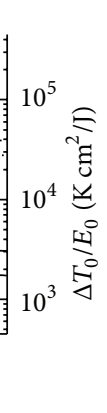

है

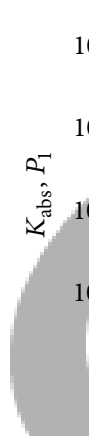

(b)
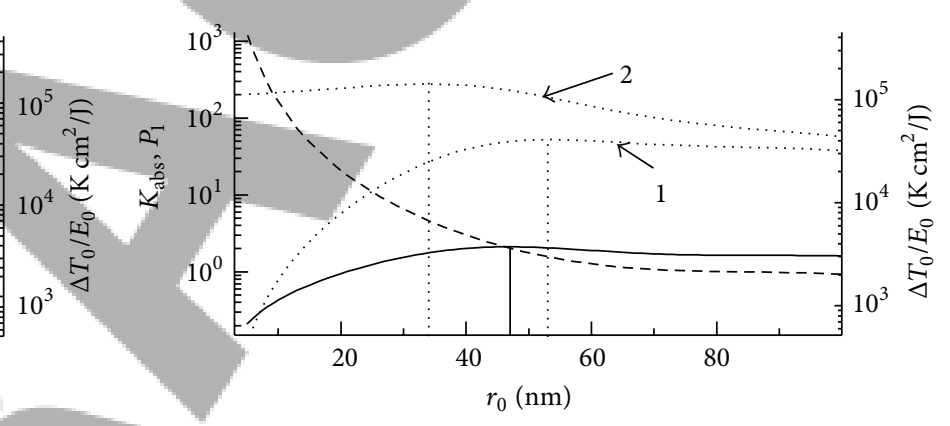

(f)

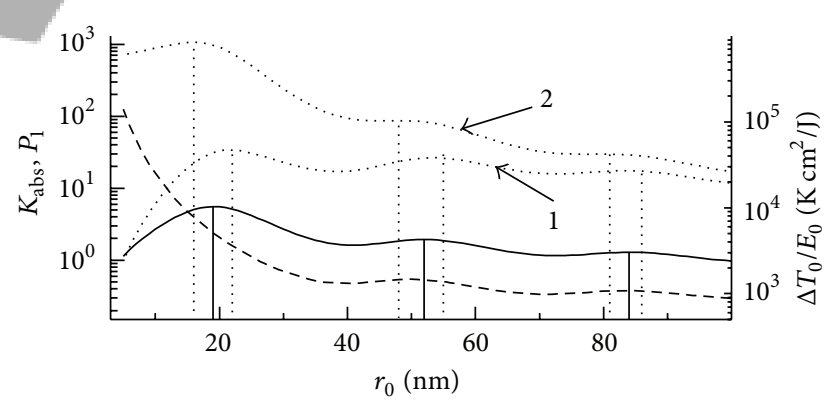

(h)

FIGURE 7: Dependences of $K_{\text {abs }}$ (solid lines refer to the left axis), parameter $P_{1}$ (dashed lines refer to the left axis), and thermooptical parameter $\Delta T_{0} / E_{0}$ (dotted lines refer to the right axis) for $t_{P}=1 \cdot 10^{-8}(1), 1 \cdot 10^{-12}(2) \mathrm{s}$ for NPs - Au, $\lambda=532 \mathrm{~nm}(\mathrm{a}), \mathrm{Ag}, \lambda=400 \mathrm{~nm}(\mathrm{~b}), \mathrm{Pt}, \lambda=500 \mathrm{~nm}$ (c), $\mathrm{Cu}, \lambda=570 \mathrm{~nm}$ (d), Pd, $\lambda=470 \mathrm{~nm}$ (e), Ti, $\lambda=500 \mathrm{~nm}$ (f), Ni, $\lambda=470 \mathrm{~nm}$ (g), and Zn, $\lambda=350 \mathrm{~nm}(\mathrm{~h})$ on $r_{0}$.

surrounding medium during radiation pulse action for pulse duration $t_{P}=1 \times 10^{-8} \mathrm{~s}$ and bigger ones.

The maximum value of $\left(\Delta T_{0} / E_{0}\right)_{\max }$ for $\mathrm{Au}$ NPs, placed in silica, is equal to $\left(\Delta T_{0} / E_{0}\right)_{\max }=5.53 \times 10^{5} \mathrm{Kcm}^{2} / \mathrm{J}$ for $r_{0}=$ $10 \mathrm{~nm}, \lambda=532 \mathrm{~nm}$, and $t_{P}=1 \times 10^{-12} \mathrm{~s}$. Maximum values for
Ag NPs, placed in different media, of about $\left(\Delta T_{0} / E_{0}\right)_{\max } \sim$ $(2-5) \times 10^{6} \mathrm{Kcm}^{2} / \mathrm{J}$ are achieved with $r_{0}=10 \mathrm{~nm}$ and $t_{P}=1 \times 10^{-12} \mathrm{~s}$ among other NPs. For example, Ag NPs with $r_{0}=10 \mathrm{~nm}$, placed in water, can achieve the heating of $\Delta T_{0}=100 \mathrm{~K}$ under action of laser pulse with $\lambda=382 \mathrm{~nm}$, 
$t_{P}=1 \times 10^{-10}, 1 \times 10^{-12} \mathrm{~s}$, and $E_{0}=5.6 \times 10^{-5} \mathrm{~J} / \mathrm{cm}^{2}$. It is connected with the achievement of maximum values of $K_{\mathrm{abs}}$ for Ag NPs in comparison with other presented NPs.

Figures 4-6 present efficiency factors of absorption $K_{\mathrm{abs}}$, scattering $K_{\text {sca }}$, and extinction $K_{\text {ext }}$ for radiation with wavelengths $\lambda$ in the spectral interval $200-1000 \mathrm{~nm}$ for homogeneous metallic spherical NPs with radii 10,25 , and $50 \mathrm{~nm}$, placed in water, for nine different metals.

Figure 4 presents factors of $K_{\mathrm{abs}}, K_{\mathrm{sca}}$, and $K_{\text {ext }}$ of radiation with wavelengths in the range $300-1000 \mathrm{~nm}$ by metallic $\mathrm{Pd}, \mathrm{Mo}$, and $\mathrm{Cu}$ NPs with radii $r_{0}=10,25$, and $50 \mathrm{~nm}$ placed in water.

The spectral dependences of $K_{\text {abs }}$ and $K_{\text {ext }}$ for Cu NPs are smooth enough for $r_{0}=10$ and $25 \mathrm{~nm}$. An interesting feature of these dependences of $K_{\mathrm{abs}}$ and $K_{\mathrm{ext}}$ on $\lambda$ for $r_{0}=10$ and $25 \mathrm{~nm}$ is the formation of so called "step" (weak dependence of $K_{\mathrm{abs}}$ and $K_{\text {ext }}$ on $\lambda$ ) for the spectral interval of $\lambda \approx$ $300-565 \mathrm{~nm}$. There is one weakly defined maximum in these curves for $310 \mathrm{~nm}\left(r_{0}=10 \mathrm{~nm}\right)$ and for $387 \mathrm{~nm}\left(r_{0}=25 \mathrm{~nm}\right)$. We see sharp folding of the dependences of $K_{\text {abs }}$ and $K_{\text {ext }}$ on $\lambda$ for $r_{0}=10$ and $25 \mathrm{~nm}$ at $\lambda \approx 565 \mathrm{~nm}$. For $r_{0}=10$ and $25 \mathrm{~nm}$ values of $K_{\mathrm{abs}} \gg K_{\mathrm{sca}}$ and dependences of $K_{\mathrm{ext}}$ and $K_{\mathrm{abs}}$ on $\lambda$ are close to each other. The factor of scattering $K_{\mathrm{sca}}$ monotonously decreases with increasing $\lambda$ for $r_{0}=10$ and $25 \mathrm{~nm}$.

In the case of $r_{0}=50 \mathrm{~nm}$ spectral dependences of $K_{\mathrm{abs}}$, $K_{\text {sca }}$, and $K_{\text {ext }}$ for $\mathrm{Cu}$ NPs have one distinct pronounced maximum: $K_{\mathrm{abs}}^{\max } \approx 2.7$ for $\lambda_{\mathrm{abs}}^{\max }=563 \mathrm{~nm}$ and $K_{\mathrm{sca}}^{\max } \approx 3.5$, $\lambda_{\text {sca }}^{\max }=590 \mathrm{~nm}$ and $K_{\text {ext }}^{\mathrm{max}} \approx 6$ for $\lambda_{\text {ext }}^{\max }=590 \mathrm{~nm}$. Positions of $K_{\mathrm{abs}}^{\max }, K_{\mathrm{sca}}^{\max }$, and $K_{\mathrm{ext}}^{\mathrm{max}}$ have been separated in Figure 4 for $r_{0}=50 \mathrm{~nm}$.

Factor $K_{\mathrm{abs}}>K_{\mathrm{sca}}$ and $P_{1}>1$ for $r_{0}=10$ and $25 \mathrm{~nm}$ and for all presented intervals of wavelengths. But for spectral interval $\lambda \approx 570-1000 \mathrm{~nm}$, the value of $P_{1}$ is smaller than 1 , $P_{1}<1$.

Maximum of $K_{\mathrm{abs}}^{\max }$ for Pd NP is shifted from the position $\lambda_{\mathrm{abs}}^{\max }=230 \mathrm{~nm}$ at $r_{0}=10 \mathrm{~nm}$ to bigger values of $\lambda$ with increasing $r_{0}$ up to $\lambda_{\mathrm{abs}}^{\max }=310 \mathrm{~nm}$ and with formation of two weakly defined maxima of $K_{\mathrm{abs}}^{\max }$ at $\lambda_{\mathrm{abs}}^{\max }=292 \mathrm{~nm}$ and $\lambda_{\mathrm{abs}}^{\max }=470 \mathrm{~nm}$ for $r_{0}=50 \mathrm{~nm}$. The maximum of $K_{\mathrm{sca}}^{\mathrm{max}}$ is shifted from the values of $\lambda_{\mathrm{sca}}^{\mathrm{max}}=190 \mathrm{~nm}$ for $r_{0}=10 \mathrm{~nm}$ to $\lambda_{\text {sca }}^{\max }=230 \mathrm{~nm}$ for $r_{0}=25 \mathrm{~nm}$, and $\lambda_{\text {sca }}^{\max }=435 \mathrm{~nm}$ at $r_{0}=50 \mathrm{~nm}$. The maximum of $K_{\mathrm{ext}}^{\mathrm{max}}$ is shifted from the values of $\lambda_{\text {ext }}^{\max }=190 \mathrm{~nm}$ for $r_{0}=10 \mathrm{~nm}$ to $\lambda_{\text {ext }}^{\max }=450 \mathrm{~nm}$ at $r_{0}=50 \mathrm{~nm}$. We see that the values of $K_{\mathrm{abs}}^{\max }, K_{\mathrm{sca}}^{\max }$, and $K_{\mathrm{ext}}^{\max }$ have been placed at different positions on $\lambda$ axis. Parameter $P_{1}$ increases up to values of $P_{1} \sim 10-100$ for $r_{0}=10,25$, and $50 \mathrm{~nm}$ with increasing of $\lambda$ in the range of $\lambda \sim 200-1000 \mathrm{~nm}$. For the spectral interval of $\lambda \sim 200-600 \mathrm{~nm}$ parameter $P_{1}$ is smaller than $1, P_{1}<1$.

Two maximum values of $\left(\Delta T_{0} / E_{0}\right)_{\max }$ are realized in Figures $4(\mathrm{c})$ and $4(\mathrm{~g})$, for $\mathrm{Cu}$ and Pd NPs because two maxima of $K_{\mathrm{abs}}^{\mathrm{max}}$ have been formed for $r_{0}=50 \mathrm{~nm}$.

For Mo NPs (Figures 4(e), 4(f), 4(g), and 4(h)) maxima of $K_{\mathrm{abs}}^{\max }$ are realized in the spectral region $180 \div 200 \mathrm{~nm}$ for $r_{0}=10$ and $25 \mathrm{~nm}$. Positions of maxima of $K_{\mathrm{sca}}^{\mathrm{max}}$ and $K_{\text {ext }}^{\max }$ are shifted from $\sim 200$ for $r_{0}=10 \mathrm{~nm}$ to $\sim 230 \mathrm{~nm}$ when increasing NP radius up to $25 \mathrm{~nm}$. When the radius is increased up to $50 \mathrm{~nm}$, two maxima of $K_{\mathrm{abs}}^{\max }, K_{\mathrm{sca}}^{\max }$, and $K_{\text {ext }}^{\max }$ are formed in all curves in Figure $4(\mathrm{~g})$. They are localized in the case of $K_{\mathrm{abs}}^{\max }$ at $\lambda_{\mathrm{abs}}^{\max }=200 \mathrm{~nm}$ and $440 \mathrm{~nm}, K_{\mathrm{sca}}^{\max }$ at $\lambda_{\text {sca }}^{\max }=230$ and $420 \mathrm{~nm}$, and $K_{\text {ext }}^{\max }$ at $\lambda_{\text {ext }}^{\max }=210$ and $420 \mathrm{~nm}$. Maximum value of absorption of Mo NPs attains $K_{\mathrm{abs}}^{\max } \approx 3.2$ for $r_{0}=10 \mathrm{~nm}$ and maximum values scattering and extinction attain $K_{\mathrm{sca}}^{\max } \approx 2.73$ and $K_{\mathrm{ext}}^{\max } \approx 4.5$ accordingly for $r_{0}=50 \mathrm{~nm}$. Two maximum values of $\left(\Delta T_{0} / E_{0}\right)_{\max }$ are realized in Figure 5(g) for Mo NPs.

Figure 5 presents spectral dependences of efficiency factors of $K_{\text {abs }}, K_{\text {sca }}$, and $K_{\text {ext }}$ of radiation in the range 150 $1000 \mathrm{~nm}$ by metallic Ni, V, and Ti NPs with radii $r_{0}=10$, 25 , and $50 \mathrm{~nm}$, placed in water. Spectral dependences of efficiency factors of $K_{\mathrm{abs}}, K_{\mathrm{sca}}$, and $K_{\text {ext }}$ for $\mathrm{Ni}, \mathrm{V}$, and Ti NPs for radii $r_{0}=10 \mathrm{~nm}$ and $25 \mathrm{~nm}$ are smooth curves with maxima in the UV region. With increasing wavelength till $1000 \mathrm{~nm}$ absorption, scattering, and extinction slowly decrease. In the case of $r_{0}=50 \mathrm{~nm}$ spectral dependences of efficiency factors of $K_{\text {abs }}$ and $K_{\text {sca }}$ for V NPs have some weakly defined maxima located both in UV and in visible region of spectra.

For Ni, V, and Ti NPs we see general features that were early noted for Figures 1-5. The first feature is the shifting of the values of $K_{\mathrm{abs}}^{\max }, K_{\mathrm{sca}}^{\max }$, and $K_{\mathrm{ext}}^{\max }$ to bigger values of $\lambda$ with increasing the NP radius; the second one is the shifting between $K_{\text {abs }}^{\max }, K_{\text {sca }}^{\max }$, and $K_{\text {ext }}^{\max }$ themselves that means that values of $\lambda_{\text {abs }}^{\max }, \lambda_{\text {sca }}^{\max }$, and $\lambda_{\text {ext }}^{\max }$ have different values, for example, for Ti NPs with $r_{0}=50 \mathrm{~nm}, \lambda_{\mathrm{abs}}^{\max }=505 \mathrm{~nm}$, $\lambda_{\text {sca }}^{\max }=355 \mathrm{~nm}$, and $\lambda_{\text {ext }}^{\max }=450 \mathrm{~nm}$. The third feature is the formation of second maximums of $K_{\mathrm{abs}}^{\max }$; for example, second maximum is formed at $\lambda \approx 480 \mathrm{~nm}$.

Parameters $P_{1}$ for Ni, $\mathrm{V}$, and Ti NPs and for the radiation spectral interval $\lambda \approx 150-1000 \mathrm{~nm}$ are bigger than $1, P_{1}>1$, instead of narrow interval $\lambda \approx 200-480 \mathrm{~nm}$ for V NPs with $r_{0}=50 \mathrm{~nm}$. Moreover, for $r_{0}=10$ and $25 \mathrm{~nm}$ parameters $P_{1}$ achieve the values of $P_{1} \approx 10-500$ with increasing $\lambda$. It means that $\mathrm{Ni}, \mathrm{Ti}$, and $\mathrm{V}$ NPs are good absorbers of radiation in wide range of ultraviolet, visible, and infrared optical spectrum.

Figure 6 presents the spectral dependences of $K_{\mathrm{abs}}, K_{\mathrm{sca}}$, and $K_{\text {ext }}$ for metallic Co, Zn, and Al NPs with radii $r_{0}=10$, 25 , and $50 \mathrm{~nm}$, when placed in water. Spectral dependences of efficiency factors of $K_{\mathrm{abs}}, K_{\mathrm{sca}}$, and $K_{\text {ext }}$ for Co nanoparticles are smooth and have some weakly defined maxima located both in UV and in visible region of spectra for $r_{0}=10$ and $25 \mathrm{~nm}$. In the case of $r_{0}=50 \mathrm{~nm}$ maximum of absorption is in the UV region of spectra and maxima of scattering and extinction are in the visible one. The maximum value of absorption for Co NPs is $K_{\mathrm{abs}}^{\max } \approx 2.5$ for $r_{0}=25 \mathrm{~nm}$, $\lambda_{\mathrm{abs}}^{\max } \approx 300 \mathrm{~nm}$, and maximum values of scattering and extinction are $K_{\mathrm{sca}}^{\max } \approx 2.2$ and $K_{\mathrm{ext}}^{\max } \approx 3.9$ for $r_{0}=50 \mathrm{~nm}$, $\lambda_{\mathrm{sca}}^{\max }=\lambda_{\mathrm{ext}}^{\max }=450 \mathrm{~nm}$.

For Zn NPs, maxima of spectral dependences of efficiency factors of $K_{\mathrm{abs}}, K_{\mathrm{sca}}$, and $K_{\text {ext }}$ are sharply defined, more than for Co NPs, and are shifted in the direction of greater wavelengths. For example, the maximum value of absorption for $\mathrm{Zn} \mathrm{NPs} \mathrm{is} K_{\mathrm{abs}}^{\max } \approx 4.5$ for $r_{0}=10 \mathrm{~nm}, \lambda_{\mathrm{abs}}^{\max } \approx 305 \mathrm{~nm}$, and maximum values of scattering and extinction are $K_{\text {sca }}^{\max } \approx 4.3$ and $K_{\mathrm{ext}}^{\mathrm{max}} \approx 5.7$ for $r_{0}=50 \mathrm{~nm}, \lambda_{\text {sca }}^{\mathrm{max}} \approx 465 \mathrm{~nm}$. We note the shifting of $K_{\mathrm{sca}}^{\mathrm{max}}$ and $K_{\mathrm{ext}}^{\mathrm{max}}$ to bigger values of $\lambda$ in comparison with position $K_{\mathrm{abs}}^{\max }$ for Co and Zn NPs. 
The spectral dependences of efficiency factors of $K_{\mathrm{abs}}$, $K_{\text {sca }}$, and $K_{\text {ext }}$ for Al NPs show strongly defined maxima located mainly in UV. Maximum values of absorption and scattering are close for $r_{0}=10 \mathrm{~nm}$, and then for $r_{0}=25$ and $50 \mathrm{~nm}$ maximum values of scattering are essentially higher than absorption. For example, maximum value of absorption for Al NPs is $K_{\mathrm{abs}}^{\max } \approx 7.5$ for $r_{0}=10 \mathrm{~nm}, \lambda_{\mathrm{abs}}^{\max } \approx 190 \mathrm{~nm}$, and maximum values of scattering and extinction are $K_{\mathrm{sca}}^{\mathrm{max}} \approx$ 6, $K_{\mathrm{ext}}^{\mathrm{max}} \approx 7$ for $r_{0}=25 \mathrm{~nm}$, and $\lambda_{\mathrm{sca}}^{\max } \approx 250 \mathrm{~nm}$. Factors of $K_{\text {sca }}$ and $K_{\text {ext }}$ rise to maximum values in the spectral interval 190-500 nm, before decreasing with increasing wavelength in the range $200-1000 \mathrm{~nm}$. Some oscillation structures of the dependences of $K_{\mathrm{abs}}$ on $\lambda$ are formed for $\mathrm{Al}$ and $\mathrm{Zn} \mathrm{NPs}$ with increasing of $r_{0}$. It is interesting to note for Al NPs the shifting of $K_{\mathrm{sca}}^{\max }$ and $K_{\mathrm{ext}}^{\mathrm{max}}$ to bigger values of $\lambda$ and the formation of two maximums of $K_{\text {sca }}^{\max }$ and $K_{\text {ext }}^{\max }$ with simultaneous formation of oscillation structure of the $K_{\text {abs }}$ dependence on $\lambda$ with increasing of $r_{0}$ to $r_{0}=50 \mathrm{~nm}$.

$\mathrm{Zn}$ NPs with $r_{0}=25 \mathrm{~nm}$ are good absorbers and bad scatterers for all spectral interval of $\lambda \approx 200-1000 \mathrm{~nm}$. The parameter $P_{1}$ for Al NPs is smaller than one $\left(P_{1}<1\right)$ for $r_{0}=$ $25 \mathrm{~nm}$ in the range of $\lambda \approx 200-500 \mathrm{~nm}$ and for $r_{0}=50 \mathrm{~nm}$, $\lambda \approx 150-1000 \mathrm{~nm}$.

Figures 1-6 present spectral dependences of the parameter $\Delta T_{0} / E_{0}$ (3) for pulse duration $t_{P}=1 \cdot 10^{-8}, 1 \cdot 10^{-12} \mathrm{~s}$ for NPs with radii $r_{0}=10,25$, and $50 \mathrm{~nm}$ placed in water. The range of pulse duration $t_{P}=1 \cdot 10^{-8}-1 \cdot 10^{-12} \mathrm{~s}$ is of great interest for laser applications in nanotechnology. The dependences of parameter $\Delta T_{0} / E_{0}$ (3) on $\lambda$ are analogous to the dependences $K_{\mathrm{abs}}(\lambda)$ because of (2a) and (2b). For $\lambda_{\mathrm{abs}}^{\max } \approx$ $190 \mathrm{~nm}$ and $r_{0}=10 \mathrm{~nm}$ maximum value of thermooptical parameter for Al NPs achieves value $\left(\Delta T_{0} / E_{0}\right)_{\max } \approx 1.5 \times$ $10^{6} \mathrm{Kcm}^{2} / \mathrm{J}$.

Figure 7 presents the dependences of $K_{\mathrm{abs}}$, parameter $P_{1}$, and thermooptical parameter $\Delta T_{0} / E_{0}$ for $t_{P}=1 \cdot 10^{-8}$, $1 \cdot 10^{-12} \mathrm{~s}$ for metallic NPs, placed in water, and fixed values of $\lambda$, for Au NPs, $\lambda=532 \mathrm{~nm}$; Ag NPs, $\lambda=400 \mathrm{~nm}$; Pt NPs, $\lambda=500 \mathrm{~nm}$; Cu NPs, $\lambda=570 \mathrm{~nm}$; Ti NPs, $\lambda=$ $500 \mathrm{~nm}$; Pd NPs, $\lambda=470 \mathrm{~nm}$; Ni NPs, $\lambda=470 \mathrm{~nm}$; and $\mathrm{Zn} \mathrm{NPs}, \lambda=350 \mathrm{~nm}$ on $r_{0}$ in the range of radii $r_{0}=$ 5-100 nm. The locations of maximum values of $K_{\mathrm{abs}}^{\max }\left(r_{0}\right)$ and $\left(\Delta T_{0} / E_{0}\right)_{\max }\left(t_{P}, r_{0}\right)$ are denoted by vertical lines (solid and dotted accordingly). The choice of mentioned wavelengths is determined by their location nearby plasmon wavelengths for these NPs (see Figures 1-6). We consider the results for $\mathrm{Au}$ and Ag NPs more closely.

Results for $\mathrm{Au}$ NPs are presented in Figure 7(a). The maximum value of $K_{\mathrm{abs}}^{\max }\left(r_{0}, \lambda\right)$ was calculated and was equal to the next value of $r_{0}: K_{\mathrm{abs}}^{\max }(\lambda=532 \mathrm{~nm}) \approx 3.97$ for $r_{0}=$ $33 \mathrm{~nm}$. The maximum values of $\left(\Delta T_{0} / E_{0}\right)_{\max }$ for $\lambda=532 \mathrm{~nm}$ are approximately equal to $\left(\Delta T_{0} / E_{0}\right)_{\max } \approx 4.1 \times 10^{5} \mathrm{Kcm}^{2} / \mathrm{J}$ at $r_{0} \approx 23 \mathrm{~nm}$ for $t_{P}=1 \cdot 10^{-12} \mathrm{~s}$ and $\left(\Delta T_{0} / E_{0}\right)_{\max } \approx 6 \times$ $10^{4} \mathrm{Kcm}^{2} / \mathrm{J}$ at $r_{0} \approx 39 \mathrm{~nm}$ for $t_{P}=1 \cdot 10^{-8} \mathrm{~s}$ (see Figure $7(\mathrm{a})$ ). The maximum values of $K_{\mathrm{abs}}^{\max }\left(r_{0}\right)$ and $\left(\Delta T_{0} / E_{0}\right)_{\max }\left(r_{0}\right)$ have different locations on $r_{0}$ axis for $\lambda=532 \mathrm{~nm}$ in Figure $7(\mathrm{a})$. The maximum values of $\left(\Delta T_{0} / E_{0}\right)_{\max }$ have been shifted by the value $\Delta r_{0} \approx 10 \mathrm{~nm}$ to smaller values of $r_{0}$ for $t_{P}=1 \cdot 10^{-12} \mathrm{~s}$ and to bigger values of $r_{0}$ by the value $\Delta r_{0} \approx 6 \mathrm{~nm}$ for $t_{P}=1 \cdot 10^{-8} \mathrm{~s}$ in comparison with the location of $K_{\mathrm{abs}}^{\mathrm{max}}\left(r_{0}\right)$ in Figure $7(\mathrm{a})$.

The maximum values of $\left(\Delta T_{0} / E_{0}\right)_{\max }$ for $\lambda=532 \mathrm{~nm}$ are achieved for $K_{\mathrm{abs}} \approx 3.3, t_{P}=1 \cdot 10^{-12} \mathrm{~s}$, and $r_{0} \approx 23 \mathrm{~nm}$ and for $K_{\mathrm{abs}} \approx 3.6, t_{P}=1 \cdot 10^{-8} \mathrm{~s}$, and $r_{0} \approx 39 \mathrm{~nm}$. It means that for achievement of the maximum values of $\left(\Delta T_{0} / E_{0}\right)_{\max }$ under minimal values of $E_{0}$ we have to use the values of $K_{\text {abs }}$ that are smaller than $K_{\mathrm{abs}}^{\max }$ mentioned above.

The differences between the values of $\Delta T_{0} / E_{0}$ for $t_{P}=1$. $10^{-8} \mathrm{~s}$ and $t_{P}=1 \cdot 10^{-12} \mathrm{~s}$ decrease with increasing $r_{0}$. These differences are about $\sim 10^{2}-10^{3}$ times for $r_{0}=10 \mathrm{~nm}$ and are equal to only $\sim 2-3$ times for $r_{0}=100 \mathrm{~nm}$. It can be explained by a sharp increase of $\tau_{T} \sim r_{0}^{2}$ and approaching of $\tau_{0}$ to $t_{P}=$ $1 \cdot 10^{-8} \mathrm{~s}$ for $r_{0} \geq 90-100 \mathrm{~nm}$ and fulfillment of short pulse condition (without heat loss).

The characteristic time $\tau_{T}$ is equal to $\tau_{T} \sim 1.2 \cdot 10^{-10}-3.2$. $10^{-9} \mathrm{~s}$ for the range $r_{0}=10-50 \mathrm{~nm}$ and for ambient water $k_{\infty}=6 \cdot 10^{-3} \mathrm{~W} / \mathrm{cmK}, \tau_{T} \sim 0.9 \mathrm{~ns}$ for $r_{0}=25 \mathrm{~nm}$. The fulfillment of the condition $t_{P}<\tau_{T}$ (1) for the most interesting range of $r_{0}: 25<r_{0}<50 \mathrm{~nm}$ means that the value of $t_{P}$ will be in the range of pulse durations: $t_{P}<1 \cdot 10^{-9} \mathrm{~s}$.

The condition of "short" pulses $t_{P}<\tau_{T}$ is applicable for $t_{P}=1 \cdot 10^{-12} \mathrm{~s}$ for all values of $r_{0}: 5<r_{0}<$ $100 \mathrm{~nm}$. Under condition of "short" pulses $t_{P}<\tau_{T}$, the parameter $\Delta T_{0} / E_{0}$ depends on the combination $K_{\mathrm{abs}} / r_{0}$ and, accordingly, equation (3) describing the increasing and decreasing of $\Delta T_{0} / E_{0}$.

The condition of "long" pulses with $t_{P}=1 \cdot 10^{-8} \mathrm{~s}$ is also fulfilled for the interval $r_{0}=5-100 \mathrm{~nm}$. The use of "long" pulses with $t_{P}=1 \cdot 10^{-8} \mathrm{~s}$ leads to a significant decrease of the value of $\Delta T_{0} / E_{0}$ up to 1-2 orders and more in comparison with cases for $t_{P}=1 \cdot 10^{-12} \mathrm{~s}$ for the whole range of $r_{0}=$ $5-100 \mathrm{~nm}$. It is determined by heat conduction losses from NP during irradiation with this value of $t_{P}$ and because of the dependence $\Delta T_{0} / E_{0} \sim 1 / t_{P}($ see $(3))$.

From (4a) and (4b) we see that

$$
\begin{gathered}
t_{P}<\tau_{T}, \quad \frac{\Delta T_{0}}{E_{0}} \sim \frac{K_{\mathrm{abs}}\left(r_{0}\right)}{r_{0}}, \\
t_{P}>\tau_{T}, \quad \frac{\Delta T_{0}}{E_{0}} \sim K_{\mathrm{abs}}\left(r_{0}\right) r_{0} .
\end{gathered}
$$

Our results are in accordance with presented dependences (5).

Figure 7(b) presents the dependences of parameter $\Delta T_{0} /$ $E_{0}\left(r_{0}, \lambda\right)(3)$ and $K_{\mathrm{abs}}\left(r_{0}, \lambda\right)$ for Ag NPs and for the pulse durations $t_{P}=1 \cdot 10^{-8}, 1 \cdot 10^{-12} \mathrm{~s}$, and $\lambda=400 \mathrm{~nm}$ on $r_{0}$. Maximum values of $\left(\Delta T_{0} / E_{0}\right)_{\max }$ for Ag NPs are equal to $\approx$ $1.1 \times 10^{6} \mathrm{Kcm}^{2} / \mathrm{J}, t_{P}=1 \cdot 10^{-12} \mathrm{~s}$, and $r_{0} \sim 19 \mathrm{~nm}$ and $\approx 5.1 \times$ $10^{4} \mathrm{Kcm}^{2} / \mathrm{J}$, and $t_{P}=1 \cdot 10^{-8} \mathrm{~s}$ at $r_{0} \sim 21 \mathrm{~nm}$ in the range 5$100 \mathrm{~nm}$ (see Figure 7(b)). Heating of NP with $r_{0} \sim 19 \mathrm{~nm}$ and for $t_{P} \leq 1 \cdot 10^{-10} \mathrm{~s}$ could achieve $1 \cdot 10^{3} \mathrm{~K}$ under radiation energy density $E_{0}=1 \times 10^{-3} \mathrm{~J} / \mathrm{cm}^{2}$.

There are three maximums of $K_{\mathrm{abs}}^{\mathrm{max}}$ and correspondingly three maximum values of $\left(\Delta T_{0} / E_{0}\right)_{\max }$ for $t_{P}=1 \times 10^{-12} \mathrm{~s}$, placed at $r_{0} \approx 19,58$, and $95 \mathrm{~nm}$, in the range of $r_{0}=$ 5-100 nm. Oscillated dependences of $\Delta T_{0} / E_{0}$ behave in an 
analogous manner to the dependences of $K_{\mathrm{abs}}$ on $r_{0}$ for the presented values of $\lambda$ (see Figure $7(\mathrm{~b})$ ). Values of $\Delta T_{0} / E_{0}$ for $t_{P}=1 \times 10^{-8} \mathrm{~s}$ are smaller than the ones for $t_{P}=1 \times 10^{-12} \mathrm{~s}$ for the whole range of $r_{0}=5-100 \mathrm{~nm}$. The values of shift between the locations of $K_{\mathrm{abs}}^{\max }$ and $\left(\Delta T_{0} / E_{0}\right)_{\max }$ for Ag NPs are smaller than in the case of Au NPs because of sharp dependences of $K_{\mathrm{abs}}$ on $r_{0}$, especially for $\lambda=400 \mathrm{~nm}$ (see Figure 7(b)).

The dependences of $K_{\mathrm{abs}}, P_{1}$, and $\Delta T_{0} / E_{0}$ on $r_{0}$ for fixed values of $\lambda$ for $\mathrm{Pt}, \mathrm{Cu}, \mathrm{Pd}, \mathrm{Ti}$, and Ni NPs are generally analogous to the dependences of Au NPs. The dependences of $\mathrm{ZnNP}$ parameters on $r_{0}$ are analogous to the dependences of Ag NPs. For all metallic NPs maximum values of $\left(\Delta T_{0} / E_{0}\right)_{\max }$ are shifted compared to location $K_{\mathrm{abs}}^{\mathrm{max}}$ in Figure $7(\mathrm{~b})$ to smaller values of $r_{0}$ for "short" pulses $t_{P}<\tau_{T}$ and to bigger values of $r_{0}$ for "long" pulses $t_{P}>\tau_{T}$.

Figures 1-6 present the dependences of $P_{1}$ on $\lambda$ for metallic NPs with radii $r_{0}=10,25$, and $50 \mathrm{~nm}$ and metals $\mathrm{Ag}, \mathrm{Al}, \mathrm{Au}, \mathrm{Co}, \mathrm{Cu}, \mathrm{Mo}, \mathrm{Ni}, \mathrm{Pd}, \mathrm{Pt}, \mathrm{Ti}, \mathrm{V}$, and $\mathrm{Zn}$. Dependences of $P_{1}$ on $\lambda$ are determined by the correlation between dependences of $K_{\mathrm{abs}}(\lambda)$ and $K_{\mathrm{sca}}(\lambda)$. Dependences of $P_{1}$ on $\lambda$ for different values of $r_{0}$ have complicated disposition. For NPs with $r_{0}=10 \mathrm{~nm}$ all presented metallic NPs exhibit high absorbance and parameter $P_{1} \gg 1$ for all metallic NPs and in part $P_{1}>1$ for some spectral intervals. The maximum values reached $P_{1} \geq 100$ for $\mathrm{Co}, \mathrm{Mo}, \mathrm{Ni}, \mathrm{Pd}, \mathrm{Pt}, \mathrm{Ti}, \mathrm{V}$, and $\mathrm{Zn}$ NPs for interval $600>\lambda>1000 \mathrm{~nm}$ and for Au and Cu NPs maximum $P_{1} \sim 100$ for $300>\lambda>500 \mathrm{~nm}$ for $r_{0}=10 \mathrm{~nm}$. All mentioned NPs are the best absorbers with $P_{1} \geq 10 \div 100$ for $600>\lambda>1000 \mathrm{~nm}$ and $r_{0}=10$ and $25 \mathrm{~nm}$ instead of $\mathrm{Au}$ and $\mathrm{Cu}$ NPs. Increasing of $r_{0}$ leads to an increase in scattering and decrease in absorbance for all presented metallic NPs. Therefore, larger NPs are more suitable for light-scattering based applications. At $r_{0}=50 \mathrm{~nm}$ instead of spectral interval $600<\lambda<1000 \mathrm{~nm}$ for Co, Mo, Ni, Pd, Ti, and Zn NPs all values of $P_{1}$ are smaller or much smaller than $1, P_{1} \ll 1$. Best scattering NPs among the studied metallic NPs are Ag NPs for $r_{0}>20 \mathrm{~nm}$. It is interesting to note that NPs can be used as absorbers in one interval of wavelengths and as scatterers in different intervals of wavelengths. All NPs with $r_{0}=25 \mathrm{~nm}$ could be the scatterers in the interval $300<\lambda<500 \mathrm{~nm}$ and the absorbers in the interval $500<\lambda<1100 \mathrm{~nm}$. Variant with value $P_{1} \approx 1$ means approximately equal possibility of using $\mathrm{NP}$ as absorber and scatterer simultaneously.

A predominant role of absorption by NP can be used for heating of NP for thermoplasmonic applications. Such NPs can be used as absorbers of radiation. A predominant role of scattering by NPs can be used for the purposes of optical diagnostics and imaging using scattered radiation. The selection of ratio between scattering and absorption with $P_{1}<1$ provides a tool for NP for contrast applications in scattering optical diagnostics.

\section{Conclusions}

The strongly enhanced absorption and scattering of spherical metallic NPs make them a novel and highly effective class of contrast agents for photothermal applications and imagingbased optical diagnostics. A number of factors need to be optimized for the success in these fields. These ones include the efficiency factors of absorption $K_{\mathrm{abs}}$, scattering $K_{\text {sca }}$, and extinction $K_{\text {ext }}$ of radiation by NP, parameters of $P_{1}$, and $\Delta T_{0} / E_{0}$. There is a need to study the dependence of these parameters on the type of metal and size of NP, radiation wavelength, parameters of surrounding medium, and so forth. Systematic study of all these characteristics is a prerequisite for the successful transition of the research promise of metallic NPs to thermal applications and has been carried out in this paper.

We conducted the investigation and analysis of plasmonic $\left(K_{\text {abs }}, K_{\text {sca }}\right.$, and $\left.K_{\text {ext }}\right)$ and thermooptical $\left(\Delta T_{0} / E_{0}\right)$ characteristics of 12 metallic NPs for radiation wavelengths in the spectral interval $200-1000 \mathrm{~nm}$ and in the range of NP radii $r_{0}=5-100 \mathrm{~nm}$, especially for $r_{0}=10,25$, and $50 \mathrm{~nm}$, based on computer and analytical modeling (Figures 1-7). Different metals were used for NPs, Au, Ag, Cu, Pt, Co, Zn, Al, Ni, Ti, V, $\mathrm{Pd}$, and Mo. Three surrounding NP media were used, silica, water, and air. Value of refractive index of surroundings in the range $n_{\lambda}=1.51-1.0$ influences the plasmonic properties with the change. The use of silica as surroundings leads to rather small deviations from the dependences with water as ambience. More pronounced deviations of NP optical and thermooptical characteristics have been determined for air surrounding.

The selection of different NPs is based on the investigation of the influence of different parameters of NP itself, radiation pulses, and ambient medium on NP properties.

The data in Figures 1-7 allow estimating the possibility to use different metallic NPs for thermoplasmonics and photonic applications. Maximum values of $K_{\text {abs }}$ were achieved for $\mathrm{Au}, \mathrm{Ag}, \mathrm{Zn}$, and $\mathrm{Cu}$. Transformation of plasmonic $\left(K_{\mathrm{abs}}\right.$, $K_{\text {sca }}$ and $\left.K_{\text {ext }}\right)$ and thermooptical $\left(\Delta T_{0} / E_{0}\right)$ properties in dependence on $\lambda, r_{0}$ with changing of NP, radiation, and ambience parameters is presented in Figures 1-7. Positions $\lambda_{\mathrm{abs}}^{\max }, \lambda_{\mathrm{sca}}^{\mathrm{max}}$, and $\lambda_{\text {ext }}^{\max }$ of maximum values of $K_{\mathrm{abs}}^{\mathrm{max}}, K_{\mathrm{sca}}^{\max }$, and $K_{\text {ext }}^{\max }$ have been determined on $\lambda$ axis and in some cases the positions of $K_{\mathrm{abs}}^{\mathrm{max}}, K_{\mathrm{sca}}^{\mathrm{max}}$, and $K_{\mathrm{ext}}^{\mathrm{max}}$ do not coincide.

Parameter of $P_{1}$ can be used for determination of the use of NP predominantly as an absorber for $P_{1}>1$ or as a scatterer for $P_{1}<1$. It is interesting to note the achievement of values of $P_{1} \geq 10-100$ for mentioned NPs with $r_{0}=10$ and $25 \mathrm{~nm}$ instead of $\mathrm{Ag}$ and Al NPs in some spectral intervals. Larger NPs are more suitable for light-scattering based applications. Best scattering NPs inside presented metallic NPs are Ag NPs for $r_{0}>20 \mathrm{~nm}$. It is interesting to note Al NPs with $r_{0}=25 \mathrm{~nm}$ which can be used as absorbers in one wavelength interval $(1100>\lambda>500 \mathrm{~nm})$ and as scatterers in the different one $(500>\lambda>300 \mathrm{~nm})$.

The main goal of light-to-thermal energy conversion and thermoplasmonics is to achieve maximum value of efficiency parameter of $\Delta T_{0} / E_{0}$ for NPs at minimal values of $E_{0}$. The influence of the parameters of radiation, $t_{P}, \lambda$, and $E_{0}$ of NP, $\rho_{0}, c_{0}, r_{0}, K_{\mathrm{abs}}$, and surrounding medium, $k_{\infty}$ and $n_{\lambda}$, reach a maximum value of $\Delta T_{0} / E_{0}$ has been established based on an analytical model. It is possible to achieve the values of about $\Delta T_{0} / E_{0} \sim 1 \cdot 10^{6} \mathrm{Kcm}^{2} / \mathrm{J}$ for NPs and for $t_{P} \leq 1 \cdot 10^{-10} \mathrm{~s}$ under radiation energy density $E_{0}=1 \cdot 10^{-3} \mathrm{~J} / \mathrm{cm}^{2}$ and the heating of such NP could achieve $1 \cdot 10^{3} \mathrm{~K}$. 
The selection of appropriate properties of NPs is based on the choice of value of $r_{0}$ for the values of determined $\lambda$ and $t_{P}$ and the choice of metallic NPs, $\lambda$, and $t_{P}$ for the determined value of $r_{0}$.

It was established that maximum values of $\Delta T_{0} / E_{0}$ and of NP temperature can be achieved with the use of the value of absorption efficiency factor $K_{\mathrm{abs}}$ smaller than maximum value of $K_{\mathrm{abs}}^{\mathrm{max}}$ taking into account irradiation duration, characteristics of NPs, and their cooling. Shift of the positions of maximum value of $\Delta T_{0} / E_{0}$ from the location of maximum value of $K_{\mathrm{abs}}^{\mathrm{max}}$ on axis $r_{0}$ is determined by noticeable influence of $r_{0}$ on the processes of NP heating and cooling.

Our results allow estimating of optimal characteristics of absorption and scattering radiation by NPs and laser energy conversion into photothermal phenomena by selection of the NP and radiation parameters and ambience properties. We present a platform for selection of the plasmonic and thermooptical properties of metallic NPs, placed in different media, for their photonic and thermoplasmonic applications.

\section{Conflict of Interests}

The authors declare that there is no conflict of interests regarding the publication of this paper.

\section{References}

[1] J. R. Adleman, D. A. Boyd, D. G. Goodwin, and D. Psaltis, "Heterogenous catalysis mediated by plasmon heating," Nano Letters, vol. 9, no. 12, pp. 4417-4423, 2009.

[2] R. Narayanan and M. A. El-Sayed, "Some aspects of colloidal nanoparticle stability, catalytic activity, and recycling potential," Topics in Catalysis, vol. 47, no. 1-2, pp. 15-21, 2008.

[3] N. J. Halas, "The photonic nanomedicine revolution: let the human side of nanotechnology emerge," Nanomedicine, vol. 4, no. 4, pp. 369-371, 2009.

[4] L. C. Kennedy, L. R. Bickford, N. A. Lewinski et al., "A new era for cancer treatment: gold-nanoparticle-mediated thermal therapies," Small, vol. 7, no. 2, pp. 169-183, 2011.

[5] X. Huang, P. K. Jain, I. H. El-Sayed, and M. A. El-Sayed, "Plasmonic photothermal therapy (PPTT) using gold nanoparticles," Lasers in Medical Science, vol. 23, no. 3, pp. 217-228, 2008.

[6] V. K. Pustovalov, A. S. Smetannikov, and V. P. Zharov, "Photothermal and accompanied phenomena of selective nanophotothermolysis with gold nanoparticles and laser pulses," Laser Physics Letters, vol. 5, no. 11, pp. 775-792, 2008.

[7] V. Pustovalov, L. Astafyeva, and B. Jean, "Computer modeling of the optical properties and heating of spherical gold and silica-gold nanoparticles for laser combined imaging and photothermal treatment," Nanotechnology, vol. 20, no. 22, Article ID 225105, 2009.

[8] V. Pustovalov, L. Astafyeva, E. Galanzha, and V. Zharov, "Thermo-optical analysis and selection of the properties of absorbing nanoparticles for laser applications in cancer nanotechnology," Cancer Nanotechnology, vol. 1, pp. 35-46, 2010.

[9] A. Csaki, F. Garwe, A. Steinbrück et al., "A parallel approach for subwavelength molecular surgery using gene-specific positioned metal nanoparticles as laser light antennas," Nano Letters, vol. 7, no. 2, pp. 247-253, 2007.
[10] J. Wang, J. D. Byrne, M. E. Napier, and J. M. Desimone, "More effective nanomedicines through particle design," Small, vol. 7, no. 14, pp. 1919-1931, 2011.

[11] S. Jain, D. Hirst, and J. O'Sullivan, "Gold nanoparticles as novel agents for cancer therapy," British Journal of Radiology, vol. 85, no. 1010, pp. 101-113, 2012.

[12] N. Zheludev, "Single nanoparticle as photonic switch and optical memory element," Journal of Optics A: Pure and Applied Optics, vol. 8, no. 4, pp. S1-S9, 2006.

[13] M. Pelton, J. Aizpurua, and G. Bryant, "Metal-nanoparticle plasmonics," Laser and Photonics Reviews, vol. 2, no. 3, pp. 136159, 2008.

[14] Y. Sonnefraud, A. L. Leen Koh, D. W. McComb, and S. A. Maier, "Nanoplasmonics: engineering and observation of localized plasmon modes," Laser and Photonics Reviews, vol. 6, no. 3, pp. 277-295, 2012.

[15] Y. Jin, Q. Li, G. Li et al., "Enhanced optical output power of blue light-emitting diodes with quasi-aligned gold nanoparticles," Nanoscale Research Letters, vol. 9, no. 1, pp. 7-13, 2014.

[16] S. Inasawa, M. Sugiyama, S. Noda, and Y. Yamaguchi, "Spectroscopic study of laser-induced phase transition of gold nanoparticles on nanosecond time scales and longer," Journal of Physical Chemistry B, vol. 110, no. 7, pp. 3114-3119, 2006.

[17] H. Muto, K. Miyajima, and F. Mafuné, "Mechanism of laserinduced size reduction of gold nanoparticles as studied by single and double laser pulse excitation," The Journal of Physical Chemistry C, vol. 112, no. 15, pp. 5810-5815, 2008.

[18] A. Pyatenko, M. Yamaguchi, and M. Suzuki, "Mechanisms of size reduction of colloidal silver and gold nanoparticles irradiated by Nd:YAG laser," Journal of Physical Chemistry C, vol. 113, no. 21, pp. 9078-9085, 2009.

[19] S. Hashimoto, D. Werner, and T. Uwada, "Studies on the interaction of pulsed lasers with plasmonic gold nanoparticles toward light manipulation, heat management, and nanofabrication," Journal of Photochemistry and Photobiology C: Photochemistry Reviews, vol. 13, no. 1, pp. 28-54, 2012.

[20] J. Wang, Y. Chen, X. Chen, J. Hao, M. Yan, and M. Qiu, "Photothermal reshaping of gold nanoparticles in a plasmonic absorber," Optics Express, vol. 19, no. 15, pp. 14726-14734, 2011.

[21] M. Honda, Y. Saito, N. I. Smith, K. Fujita, and S. Kawata, "Nanoscale heating of laser irradiated single gold nanoparticles in liquid," Optics Express, vol. 19, no. 13, pp. 12375-12383, 2011.

[22] A. L. Stepanov, "Nonlinear optical properties of implanted metal nanoparticles in various transparent matrixes: a review," Reviews on Advanced Materials Science, vol. 27, no. 2, pp. 115145, 2011.

[23] A. Stalmashonak, G. Seifert, and A. Abdolvand, Ultra-Short Pulsed Laser Engineered Metal-Glass Nanocomposites, Springer, New York, NY, USA, 2013.

[24] V. K. Pustovalov, "Theoretical study of heating of spherical nanoparticle in media by short laser pulses," Chemical Physics, vol. 308, no. 1-2, pp. 103-109, 2005.

[25] A. O. Govorov and H. H. Richardson, "Generating heat with metal nanoparticles," Nano Today, vol. 2, no. 1, pp. 30-38, 2007.

[26] V. K. Pustovalov, L. G. Astafyeva, and W. Fritzsche, "Selection of thermo-optical parameter of nanoparticles for achievement of their maximal thermal energy under optical irradiation," Nano Energy, vol. 2, no. 6, pp. 1137-1141, 2013.

[27] G. Baffou and R. Quidant, "Thermo-plasmonics: using metallic nanostructures as nano-sources of heat," Laser and Photonics Reviews, vol. 7, no. 2, pp. 171-187, 2013. 
[28] U. Kreibig and M. Vollmer, Optical Properties of Metal Clusters, vol. 25 of Springer Series in Material Science, Springer, Heidelberg, Germany, 1995.

[29] C. F. Bohren and D. R. Huffman, Absorption and Scattering of Light by Small Particles, Wiley, New York, NY, USA, 1983.

[30] V. K. Pustovalov and V. A. Babenko, "Optical properties of gold nanoparticles at laser radiation wavelengths for laser applications in nanotechnology and medicine," Laser Physics Letters, vol. 1, no. 10, pp. 516-520, 2004.

[31] P. K. Jain, X. Huang, I. H. El-Sayed, and M. A. El-Sayed, "Review of some interesting surface plasmon resonance-enhanced properties of noble metal nanoparticles and their applications to biosystems," Plasmonics, vol. 2, no. 3, pp. 107-118, 2007.

[32] M. G. Blaber, M. D. Arnold, and M. J. Ford, "Search for the ideal lasmonic nanoshell: the effects of surface scattering and alternatives to gold and silver," Journal of Physical Chemistry C, vol. 113, no. 8, pp. 3041-3045, 2009.

[33] V. Amendola, O. M. Bakr, and F. Stellacci, "A study of the surface plasmon resonance of silver nanoparticles by the discrete dipole approximation method: effect of shape, size, structure, and assembly," Plasmonics, vol. 5, no. 1, pp. 85-97, 2010.

[34] S. A. Joseph, S. Mathew, G. Sharma et al., "Phototermal characterization of nanogold under conditions of resonant excitation and energy transfer," Plasmonics, vol. 5, no. 1, pp. 63-68, 2010.

[35] P. R. West, S. Ishii, G. V. Naik, N. K. Emani, V. M. Shalaev, and A. Boltasseva, "Searching for better plasmonic materials," Laser and Photonics Reviews, vol. 4, no. 6, pp. 795-808, 2010.

[36] Y. Sonnefraud, A. Koh, D. W. McComb, and S. A. Maier, "Nanoplasmonics: engineering and observation of localized plasmon modes," Laser and Photonics Reviews, vol. 6, no. 3, pp. 277-295, 2012.

[37] A. Chen and P. Holt-Hindle, "Platinum-based nanostructured materials: synthesis, properties, and applications," Chemical Reviews, vol. 110, no. 6, pp. 3767-3804, 2010.

[38] M. B. Cortie and A. M. McDonagh, "Synthesis and optical properties of hybrid and alloy plasmonic nanoparticles," Chemical Reviews, vol. 111, no. 6, pp. 3713-3735, 2011.

[39] M. Rycenga, C. M. Cobley, J. Zeng et al., "Controlling the synthesis and assembly of silver nanostructures for plasmonic applications," Chemical Reviews, vol. 111, no. 6, pp. 3669-3712, 2011.

[40] P. B. Johnson and R. W. Christy, "Optical constants of the noble metals," Physical Review B, vol. 6, no. 12, pp. 4370-4379, 1972.

[41] E. D. Palik, Handbook of Optical Constants of Solids, Academic Press, New York, NY, USA, 1998.

[42] SOPRA N \& K database [Electronic resource], http://refractiveindex.info/.

[43] E. Grigor'ev and E. Meilikhov, Physical Quantities, Atomizdat, Moscow, Russia, 1991.

[44] F. Kreith and W. Z. Black, Basic Heat Transfer, Harper and Row, New York, NY, USA, 1980.

[45] N. C. Bigall, T. Härtling, M. Klose, P. Simon, L. M. Eng, and A. Eychmüller, "Monodisperse platinum nanospheres with adjustable diameters from 10 to $100 \mathrm{~nm}$ : synthesis and distinct optical properties," Nano Letters, vol. 8, no. 12, pp. 4588-4592, 2008.

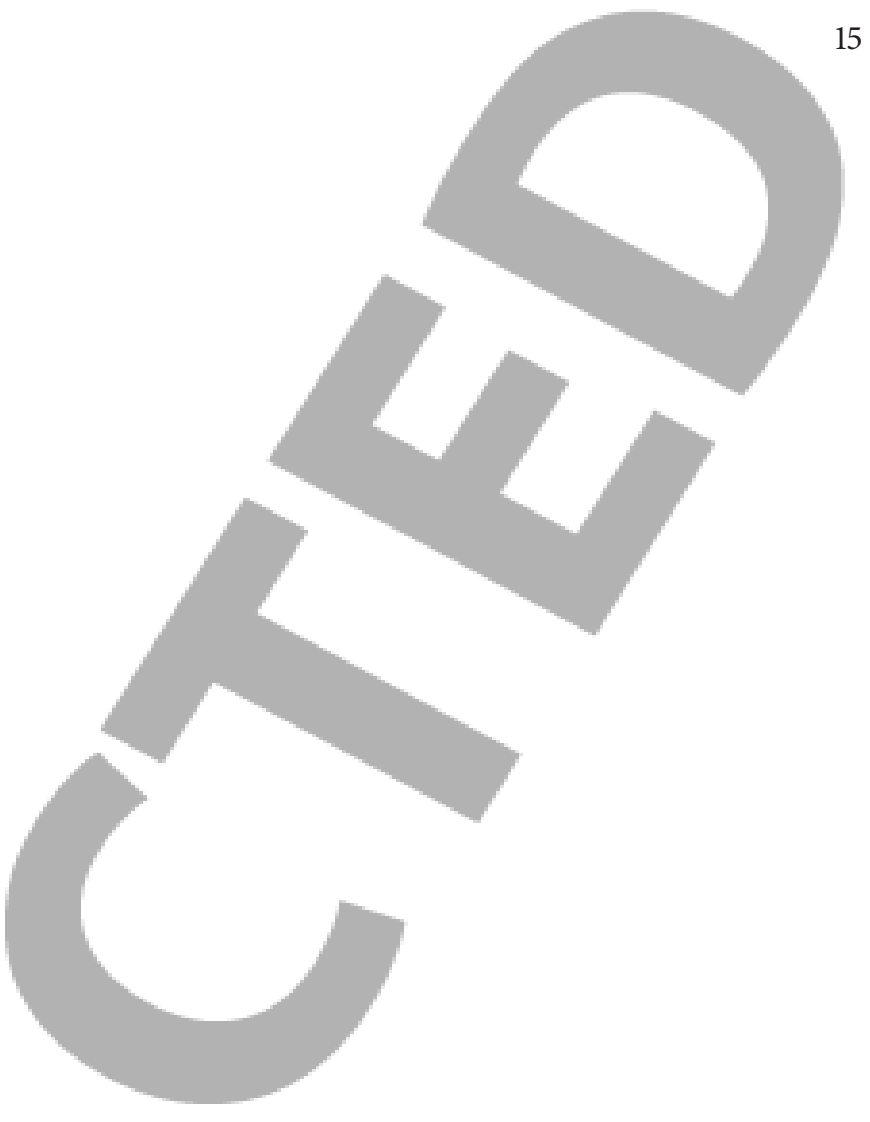

\section{(}

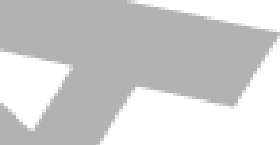

